Pacific

Journal of

Mathematics

FAMILIES AND SPRINGER'S CORRESPONDENCE

George LusZTig

Volume 267 No. 2

February 2014 


\title{
FAMILIES AND SPRINGER'S CORRESPONDENCE
}

\author{
GEORGE LUSZTIG
}

\begin{abstract}
We establish a relationship between the known parametrization of a family of irreducible representations of a Weyl group and Springer's correspondence.
\end{abstract}

\section{Introduction}

0.1. Let $G$ be a connected reductive algebraic group over an algebraically closed field $\mathbb{k}$ of characteristic $p$. Let $W$ be the Weyl group of $G$; let $\operatorname{Irr} W$ be a set of representatives for the isomorphism classes of irreducible representations of $W$ over $\overline{\mathbb{Q}}_{l}$, an algebraic closure of the field of $l$-adic numbers ( $l$ is a fixed prime number other than $p$ ).

Now Irr $W$ is partitioned into subsets called families as in [Lusztig 1979b, $\S 9$; 1984a, 4.2]. Moreover to each family $\mathscr{F}$ in $\operatorname{Irr} W$, a certain set $\boldsymbol{X}_{\mathscr{F}}$, a pairing $\{\}:, \boldsymbol{X}_{\mathscr{F}} \times \boldsymbol{X}_{\mathscr{F}} \rightarrow \overline{\mathbb{Q}}_{l}$, and an imbedding $\mathscr{F} \rightarrow \boldsymbol{X}_{\mathscr{F}}$ was canonically attached in [Lusztig 1979b; 1984a, Chapter 4]. (The set $\boldsymbol{X}_{\mathscr{F}}$ with the pairing \{, \}, which can be viewed as a nonabelian analogue of a symplectic vector space, plays a key role in the classification of unipotent representations of a finite Chevalley group [Lusztig 1984a] and in that of unipotent character sheaves on G.) In [Lusztig 1979b; 1984a] it is shown that $\boldsymbol{X}_{\mathscr{F}}=M\left(\mathscr{G}_{\mathscr{F}}\right)$ where $\mathscr{G}_{\mathscr{F}}$ is a certain finite group associated to $\mathscr{F}$ and, for any finite group $\Gamma, M(\Gamma)$ is the set of all pairs $(g, \rho)$ where $g$ is an element of $\Gamma$ defined up to conjugacy and $\rho$ is an irreducible representation over $\overline{\mathbb{Q}}_{l}$ (up to isomorphism) of the centralizer of $g$ in $\Gamma$; moreover $\{$,$\} is given by the$ "nonabelian Fourier transform matrix" of [Lusztig 1979b, § 4] for $\mathscr{G}_{\mathscr{F}}$.

In the remainder of this paper we assume that $p$ is not a bad prime for $G$. In this case a uniform definition of the group $\mathscr{G}_{\mathscr{F}}$ was proposed in [Lusztig 1984a, 13.1] in terms of special unipotent classes in $G$ and the Springer correspondence, but the fact that this leads to a group isomorphic to $\mathscr{G}_{\mathscr{F}}$ as defined in [Lusztig 1984a, Chapter 4] was stated in [Lusztig 1984a, (13.1.3)] without proof. One of the aims of this paper is to supply the missing proof.

Supported in part by National Science Foundation grant DMS-0758262.

MSC2010: 20G99.

Keywords: Weyl group, unipotent class, Springer correspondence. 
To state the results of this paper we need some definitions. For $E \in \operatorname{Irr} W$ let $a_{E} \in \mathbb{N}, b_{E} \in \mathbb{N}$ be as in [Lusztig 1984a, 4.1]. As noted in [Lusztig 1979a], for $E \in \operatorname{Irr} W$ we have

$$
a_{E} \leq b_{E}
$$

we say that $E$ is special if $a_{E}=b_{E}$.

For $g \in G$ let $Z_{G}(g)$ or $Z(g)$ be the centralizer of $g$ in $G$ and let $A_{G}(g)$ or $A(g)$ be the group of connected components of $Z(g)$. Let $C$ be a unipotent conjugacy class in $G$ and let $u \in C$. Let $\mathscr{B}_{u}$ be the variety of Borel subgroups of $G$ that contain $u$; this is a nonempty variety of dimension, say, $e_{C}$. The conjugation action of $Z(u)$ on $\mathscr{B}_{u}$ induces an action of $A(u)$ on $\boldsymbol{S}_{u}:=H^{2 e_{C}}\left(\mathscr{P}_{u}, \overline{\mathbb{Q}}_{l}\right)$. Now $W$ acts on $\boldsymbol{S}_{u}$ by Springer's representation [Springer 1976]; however here we adopt the definition of the $W$-action on $S_{u}$ given in [Lusztig 1984b] which differs from Springer's original definition by tensoring by sign. The $W$-action on $S_{u}$ commutes with the $A(u)$-action. Hence we have canonically $\boldsymbol{S}_{u}=\oplus_{E \in \operatorname{Irr} W} E \otimes \mathscr{V}_{E}$ (as $W \times A(u)$ modules) where $\mathscr{V}_{E}$ are finite dimensional $\overline{\mathbb{Q}}_{l}$-vector spaces with $A(u)$-action. Let $\operatorname{Irr}_{C} W=\left\{E \in \operatorname{Irr} W ; \mathscr{V}_{E} \neq 0\right\}$; this set does not depend on the choice of $u$ in $C$. By [Springer 1976], the sets $\operatorname{Irr}_{C} W$ (for $C$ variable) form a partition of $\operatorname{Irr} W$; also, if $E \in \operatorname{Irr}_{C} W$ then $\mathscr{V}_{E}$ is an irreducible $A(u)$-module and, if $E \neq E^{\prime} \operatorname{in}^{\operatorname{Irr}_{C}} W$, then the $A(u)$-modules $\mathscr{V}_{E}, \mathscr{V}_{E^{\prime}}$ are not isomorphic. By [Borho and MacPherson 1981] we have

$$
e_{C} \leq b_{E} \quad \text { for any } E \in \operatorname{Irr}_{C} W \text {, }
$$

and the equality $b_{E}=e_{C}$ holds for exactly one $E \in \operatorname{Irr}_{C} W$ which we denote by $E_{C}$ (for this $E, \mathscr{V}_{E}$ is the unit representation of $A(u)$ ).

Following [Lusztig 1984a, (13.1.1)] we say that $C$ is special if $E_{C}$ is special. (This concept was introduced in [Lusztig 1979a, § 9] although the word "special" was not used there.) From (b) we see that $C$ is special if and only if $a_{E_{C}}=e_{C}$.

Now assume that $C$ is special. We denote by $\mathscr{F} \subset \operatorname{Irr} W$ the family that contains $E_{C}$. (Note that $C \mapsto \mathscr{F}$ is a bijection from the set of special unipotent classes in $G$ to the set of families in $\operatorname{Irr} W$.) We set $\operatorname{Irr}_{C}^{*} W=\left\{E \in \operatorname{Irr}_{C} W ; E \in \mathscr{F}\right\}$ and

$$
\mathscr{K}(u)=\left\{a \in A(u) ; a \text { acts trivially on } \mathscr{V}_{E} \text { for any } E \in \operatorname{Irr}_{C}^{*} W\right\}
$$

This is a normal subgroup of $A(u)$. We set $\bar{A}(u)=A(u) / \mathscr{K}(u)$, a quotient group of $A(u)$. Now, for any $E \in \operatorname{Irr}_{C}^{*} W, \mathscr{V}_{E}$ is naturally an (irreducible) $\bar{A}(u)$-module. Another definition of $\bar{A}(u)$ is given in [Lusztig 1984a, (13.1.1)]. In that definition $\operatorname{Irr}_{C}^{*} W$ is replaced by $\left\{E \in \operatorname{Irr}_{C} W ; a_{E}=e_{C}\right\}$ and $\mathscr{K}(u), \bar{A}(u)$ are defined as above but in terms of this modified $\operatorname{Irr}_{C}^{*} W$. However the two definitions are equivalent in view of the following result. 
Proposition 0.2. Assume that $C$ is special. Let $E \in \operatorname{Irr}_{C} W$.

(a) We have $a_{E} \leq e_{C}$.

(b) We have $a_{E}=e_{C}$ if and only if $E \in \mathscr{F}$.

This follows from [Lusztig 1992, 10.9]. Note that (a) was stated without proof in [Lusztig 1984a, (13.1.2)] (the proof I had in mind at the time of [Lusztig 1984a] was combinatorial).

0.3. The following result is equivalent to a result stated without proof in [Lusztig 1984a, (13.1.3)].

Theorem 0.4. Let $C$ be a special unipotent class of $G$, let $u \in C$ and let $\mathscr{F}$ be the family that contains $E_{C}$. Then we have canonically $\boldsymbol{X}_{\mathscr{F}}=M(\bar{A}(u))$ so that the pairing $\{$,$\} on \boldsymbol{X}_{\mathscr{F}}$ coincides with the pairing $\{$,$\} on M(\bar{A}(u))$. Hence $\mathscr{G}_{\mathscr{F}}$ can be taken to be $\bar{A}(u)$.

This is equivalent to the corresponding statement in the case where $G$ is adjoint, which reduces immediately to the case where $G$ is adjoint simple. It is then enough to prove the theorem for one $G$ in each isogeny class of semisimple, almost simple algebraic groups; this will be done in Section 3 after some combinatorial preliminaries in Sections 1 and 2. The proof uses the explicit description of the Springer correspondence: for type $A_{n}, G_{2}$ in [Springer 1976]; for type $B_{n}, C_{n}, D_{n}$ in [Shoji 1979a; 1979b] (as an algorithm) and in [Lusztig 1984b] (by a closed formula); for type $F_{4}$ in [Shoji 1980]; for type $E_{n}$ in [Alvis and Lusztig 1982; Spaltenstein 1982].

An immediate consequence of (the proof of) Theorem 0.4 is the following result which answers a question of R. Bezrukavnikov and which plays a role in [Losev and Ostrik 2012].

Corollary 0.5. In the setup of Theorem 0.4 let $E \in \operatorname{Irr}_{C}^{*} W$ and let $\mathscr{V}_{E}$ be the corresponding $A(u)$-module viewed as an (irreducible) $\bar{A}(u)$-module. The image of $E$ under the canonical imbedding $\mathscr{F}_{F} \rightarrow \boldsymbol{X}_{\mathscr{F}}=M(\bar{A}(u))$ is represented by the pair $\left(1, \mathscr{V}_{E}\right) \in M(\bar{A}(u))$. Conversely, if $E \in \mathscr{F}_{F}$ and the image of $E$ under $\mathscr{F}_{F} \rightarrow \boldsymbol{X}_{\mathscr{F}}=$ $M(\bar{A}(u))$ is represented by the pair $(1, \rho) \in M(\bar{A}(u))$ where $\rho$ is an irreducible representation of $\bar{A}(u)$, then $E \in \operatorname{Irr}_{C}^{*} W$ and $\rho \cong \mathscr{V}_{E}$.

0.6. Corollary 0.5 has the following interpretation. Let $Y$ be a (unipotent) character sheaf on $G$ whose restriction to the regular semisimple elements is $\neq 0$; assume that in the usual parametrization of unipotent character sheaves by $\bigsqcup_{\mathscr{F}^{\prime}} \boldsymbol{X}_{\mathscr{F}^{\prime}}, Y$ corresponds to $(1, \rho) \in M(\bar{A}(u))$ where $C$ is the special unipotent class corresponding to a family $\mathscr{F}, u \in C$ and $\rho$ is an irreducible representation of $\bar{A}(u)$. Then $\left.Y\right|_{C}$ is (up to shift) the irreducible local system on $C$ defined by $\rho$. 
0.7. Notation. If $A, B$ are subsets of $\mathbb{N}$ we denote by $A \cup \dot{\cup} B$ the union of $A$ and $B$ regarded as a multiset (each element of $A \cap B$ appears twice). For any set $\mathscr{X}$, we denote by $\mathscr{P}(\mathscr{X})$ the set of subsets of $\mathscr{X}$ viewed as an $F_{2}$-vector space with sum given by the symmetric difference. If $\mathscr{X} \neq \varnothing$ we note that $\{\varnothing, \mathscr{X}\}$ is a line in $\mathscr{P}(\mathscr{X})$ and we set $\overline{\mathscr{P}}(\mathscr{X})=\mathscr{P}(\mathscr{X}) /\{\varnothing, \mathscr{X}\}, \mathscr{P}_{\mathrm{ev}}(\mathscr{X})=\{L \in \mathscr{P}(\mathscr{X}) ;|L|=0 \bmod 2\}$; let $\overline{\mathscr{P}}_{\mathrm{ev}}(\mathscr{X})$ be the image of $\mathscr{P}_{\mathrm{ev}}(\mathscr{X})$ under the obvious map $\mathscr{P}(\mathscr{X}) \rightarrow \overline{\mathscr{P}}(\mathscr{X})$ (thus $\overline{\mathscr{P}}_{\mathrm{ev}}(\mathscr{X})=\overline{\mathscr{P}}(\mathscr{X})$ if $|\mathscr{X}|$ is odd and $\overline{\mathscr{P}}_{\mathrm{ev}}(\mathscr{X})$ is a hyperplane in $\overline{\mathscr{P}}(\mathscr{X})$ if $|\mathscr{X}|$ is even). Now if $\mathscr{X} \neq \varnothing$, the assignment $L, L^{\prime} \mapsto\left|L \cap L^{\prime}\right| \bmod 2$ defines a symplectic form on $\mathscr{P}_{\mathrm{ev}}(\mathscr{C})$ which induces a nondegenerate symplectic form $\left(\right.$, , ) on $\overline{\mathscr{P}}_{\mathrm{ev}}(\mathscr{C})$ via the obvious linear $\operatorname{map} \mathscr{P}_{\mathrm{ev}}(\mathscr{X}) \rightarrow \overline{\mathscr{P}}_{\mathrm{ev}}(\mathscr{X})$.

For $g \in G$ let $g_{s}$ and $g_{\omega}$ be the semisimple and unipotent parts of $g$.

For $z \in \frac{1}{2} \mathbb{Z}$ we set $\lfloor z\rfloor=z$ if $z \in \mathbb{Z}$ and $\lfloor z\rfloor=z-\frac{1}{2}$ if $z \in \mathbb{Z}+\frac{1}{2}$.

Errata to [Lusztig 1984a]. On page 86 , on line -6 delete " $b$ ' $<b$ " and on line -4 before "In the language..." insert "The array above is regarded as identical to the array obtained by interchanging its two rows."

On page 343 , line -5 , after "respect to $M$ " insert "and where the group $\mathscr{G}_{\mathscr{F}}$ defined in terms of $\left(u^{\prime}, M\right)$ is isomorphic to the group $\mathscr{G}_{\mathscr{F}}$ defined in terms of $(u, G)$ ”.

Erratum to [Lusztig 1984b]. In the definition of $A_{\alpha}, B_{\alpha}$ in [Lusztig 1984b, 11.5], the condition $I \in \alpha$ should be replaced by $I \in \alpha^{\prime}$ and the condition $I \in \alpha^{\prime}$ should be replaced by $I \in \alpha$.

\section{Combinatorics}

1.1. Let $N$ be an even integer $\geq 0$. Let $a:=\left(a_{0}, a_{1}, a_{2}, \ldots, a_{N}\right) \in \mathbb{N}^{N+1}$ be such that $a_{0} \leq a_{1} \leq a_{2} \leq \cdots \leq a_{N}, a_{0}<a_{2}<a_{4}<\cdots, a_{1}<a_{3}<a_{5}<\cdots$. Let $\mathscr{F}=\left\{i \in[0, N] ; a_{i}\right.$ appears exactly once in $\left.a\right\}$. We have $\mathscr{F}=\left\{i_{0}, i_{1}, \ldots, i_{2 M}\right\}$ where $M \in \mathbb{N}$ and $i_{0}<i_{1}<\cdots<i_{2 M}$ satisfy $i_{s}=s \bmod 2$ for $s \in[0,2 M]$. Hence for any $s \in[0,2 M-1]$ we have $i_{s+1}=i_{s}+2 m_{s}+1$ for some $m_{s} \in \mathbb{N}$. Let $\mathscr{E}$ be the set of $b:=\left(b_{0}, b_{1}, b_{2}, \ldots, b_{N}\right) \in \mathbb{N}^{N+1}$ such that $b_{0}<b_{2}<b_{4}<\cdots$, $b_{1}<b_{3}<b_{5}<\cdots$ and such that $[b]=[a]$ (we denote by $[b],[a]$ the multisets $\left.\left\{b_{0}, b_{1}, \ldots, b_{N}\right\},\left\{a_{0}, a_{1}, \ldots, a_{N}\right\}\right)$. We have $a \in \mathscr{E}$. For $b \in \mathscr{E}$ we set

$$
\begin{aligned}
\hat{b} & =\left(\hat{b}_{0}, \hat{b}_{1}, \hat{b}_{2}, \ldots, \hat{b}_{N}\right) \\
& =\left(b_{0}, b_{1}+1, b_{2}+1, b_{3}+2, b_{4}+2, \ldots, b_{N-1}+N / 2, b_{N}+N / 2\right) .
\end{aligned}
$$

Let $[\hat{b}]$ be the multiset $\left\{\hat{b}_{0}, \hat{b}_{1}, \hat{b}_{2}, \ldots, \hat{b}_{N}\right\}$. For $s \in\{1,3, \ldots, 2 M-1\}$ we define $a^{\{s\}}=\left(a_{0}^{\{s\}}, a_{1}^{\{s\}}, a_{2}^{\{s\}}, \ldots, a_{N}^{\{s\}}\right) \in \mathscr{E}$ by

$$
\begin{array}{r}
\left(a_{i_{s}}^{\{s\}}, a_{i_{s}+1}^{\{s\}}, a_{i_{s}+2}^{\{s\}}, a_{i_{s}+3}^{\{s\}}, \ldots, a_{i_{s}+2 m_{s}}^{\{s\}}, a_{i_{s}+2 m_{s}+1}^{\{s\}}\right) \\
\quad=\left(a_{i_{s}+1}, a_{i_{s}}, a_{i_{s}+3}, a_{i_{s}+2}, \ldots, a_{i_{s}+2 m_{s}+1}, a_{i_{s}+2 m_{s}}\right)
\end{array}
$$


and $a_{i}^{\{s\}}=a_{i}$ if $i \in[0, N]-\left[i_{s}, i_{s+1}\right]$. More generally, for $X \subset\{1,3, \ldots, 2 M-1\}$ we define $a^{X}=\left(a_{0}^{X}, a_{1}^{X}, a_{2}^{X}, \ldots, a_{N}^{X}\right) \in \mathscr{E}$ by $a_{i}^{X}=a_{i}^{\{s\}}$ if $s \in X, i \in\left[i_{s}, i_{s+1}\right]$, and $a_{i}^{X}=a_{i}$ for all other $i \in[0, N]$. Note that $\left[\widehat{a^{X}}\right]=[\hat{a}]$. Conversely, we have the following result.

Lemma 1.2. Let $b \in \mathscr{E}$ be such that $[\hat{b}]=[\hat{a}]$. There exists $X \subset\{1,3, \ldots, 2 M-1\}$ such that $b=a^{X}$.

The proof is given in 1.3-1.5.

1.3. We argue by induction on $M$. We have

$$
a=\left(y_{1}=y_{1}<y_{2}=y_{2}<\cdots<y_{r}=y_{r}<a_{i_{0}}<\ldots\right)
$$

for some $r$. Since $[b]=[a]$, we must have

$$
\left(b_{0}, b_{2}, b_{4}, \ldots\right)=\left(y_{1}, y_{2}, \ldots, y_{r}, \ldots\right),\left(b_{1}, b_{3}, b_{5}, \ldots\right)=\left(y_{1}, y_{2}, \ldots, y_{r}, \ldots\right) .
$$

Thus,

$$
b_{i}=a_{i} \text { for } i<i_{0} .
$$

We have $a=\left(\cdots<a_{i_{2 M}}<y_{1}^{\prime}=y_{1}^{\prime}<y_{2}^{\prime}=y_{2}^{\prime}<\cdots<y_{r^{\prime}}^{\prime}=y_{r^{\prime}}^{\prime}\right)$ for some $r^{\prime}$. Since $[b]=[a]$, we must have

$$
\left(b_{0}, b_{2}, b_{4}, \ldots\right)=\left(\ldots, y_{1}^{\prime}, y_{2}^{\prime}, \ldots, y_{r^{\prime}}^{\prime}\right),\left(b_{1}, b_{3}, b_{5}, \ldots\right)=\left(\ldots, y_{1}^{\prime}, y_{2}^{\prime}, \ldots, y_{r^{\prime}}^{\prime}\right) \text {. }
$$

Thus,

$$
b_{i}=a_{i} \quad \text { for } i>i_{2 M} .
$$

If $M=0$ we see that $b=a$ and there is nothing further to prove. In the rest of the proof we assume that $M \geq 1$.

1.4. From 1.3 we see that

$$
\left(a_{0}, a_{1}, a_{2}, \ldots, a_{i_{2 M}}\right)=\left(\ldots, a_{i_{2 M-1}}<x_{1}=x_{1}<x_{2}=x_{2}<\cdots<x_{q}=x_{q}<a_{i_{2 M}}\right)
$$

(for some $q$ ) has the same entries as $\left(b_{0}, b_{1}, b_{2}, \ldots, b_{i_{2 M}}\right)$ (in some order). Hence the pair

$$
\left(\ldots, b_{i_{2 M}-5}, b_{i_{2 M}-3}, b_{i_{2 M-1}}\right),\left(\ldots, b_{i_{2 M}-4}, b_{i_{2 M}-2}, b_{i_{2 M}}\right)
$$

must have one of the following four forms.

$$
\begin{aligned}
& \left(\ldots, a_{i_{2 M-1}}, x_{1}, x_{2}, \ldots, x_{q}\right),\left(\ldots, x_{1}, x_{2}, \ldots, x_{q}, a_{i_{2 M}}\right), \\
& \left(\ldots, x_{1}, x_{2}, \ldots, x_{q}, a_{i_{2 M}}\right),\left(\ldots, a_{i_{2 M-1}}, x_{1}, x_{2}, \ldots, x_{q}\right), \\
& \left(\ldots, x_{1}, x_{2}, \ldots, x_{q}\right),\left(\ldots, a_{i_{2 M-1}}, x_{1}, x_{2}, \ldots, x_{q}, a_{i_{2 M}}\right), \\
& \left(\ldots, a_{i_{2 M-1}}, x_{1}, x_{2}, \ldots, x_{q}, a_{i_{2 M}}\right),\left(\ldots, x_{1}, x_{2}, \ldots, x_{q}\right) .
\end{aligned}
$$


Hence $\left(\ldots, b_{i_{2 M}-2}, b_{i_{2 M}-1}, b_{i_{2 M}}\right)$ must have one of the following four forms.

(I) $\left(\ldots, a_{i_{2 M-1}}, x_{1}, x_{1}, x_{2}, x_{2}, \ldots, x_{q}, x_{q}, a_{i_{2 M}}\right)$,

(II) $\left(\ldots, x_{1}, a_{i_{2 M-1}}, x_{2}, x_{1}, x_{3}, x_{2}, \ldots, x_{q}, x_{q-1}, a_{i_{2 M}}, x_{q}\right)$,

(III) $\left(\ldots, a_{i_{2 M-1}}, z, x_{1}, x_{1}, x_{2}, x_{2}, \ldots, x_{q}, x_{q}, a_{i_{2 M}}\right)$,

(IV) $\left(\ldots, a_{i_{2 M-1}}, z^{\prime}, x_{1}, z^{\prime \prime}, x_{2}, x_{1}, x_{3}, x_{2}, \ldots, x_{q}, x_{q-1}, a_{i_{2 M}}, x_{q}\right)$,

where $a_{i_{2 M-1}}>z, a_{i_{2 M-1}}>z^{\prime \prime} \geq z^{\prime}$ and all entries in ... are $<a_{i_{2 M-1}}$. Correspondingly, $\left(\ldots, \hat{b}_{i_{2 M}-2}, \hat{b}_{i_{2 M}-1}, \hat{b}_{i_{2 M}}\right)$ must have one of the following four forms.

(I) $\left(. ., a_{i_{2 M-1}}+h-q, x_{1}+h-q, x_{1}+h-q+1, x_{2}+h-q+1, x_{2}+h-q+2\right.$, $\left.\ldots, x_{q}+h-1, x_{q}+h, a_{i_{2 M}}+h\right)$,

(II) $\left(. ., x_{1}+h-q, a_{i_{2 M-1}}+h-q, x_{2}+h-q+1, x_{1}+h-q+1, x_{3}+h-q+2\right.$, $\left.x_{2}+h-q+1, \ldots, x_{q}+h-1, x_{q-1}+h-1, a_{i_{2 M}}+h, x_{q}+h\right)$,

(III) $\left(. ., a_{i_{2 M-1}}+h-q-1, z+h-q, x_{1}+h-q, x_{1}+h-q+1, x_{2}+h-q+1\right.$, $\left.x_{2}+h-q+2, \ldots, x_{q}+h-1, x_{q}+h, a_{i_{2 M}}+h\right)$,

(IV) $\left(. ., a_{i_{2 M-1}}+h-q-1, z^{\prime}+h-q-1, x_{1}+h-q, z^{\prime \prime}+h-q, x_{2}+h-q+1\right.$, $x_{1}+h-q+1, x_{3}+h-q+2, x_{2}+h-q+1, \ldots, x_{q}+h-1, x_{q-1}+h-1$, $\left.a_{i_{2 M}}+h, x_{q}+h\right)$,

where $h=i_{2 M} / 2$ and in cases (III) and (IV), $a_{i_{2 M-1}}+h-q$ is not an entry of $\left(\ldots, \hat{b}_{i_{2 M}-2}, \hat{b}_{i_{2 M}-1}, \hat{b}_{i_{2 M}}\right)$.

Since $\left(\ldots, \hat{a}_{i_{2 M}-2}, \hat{a}_{i_{2 M}-1}, \hat{a}_{i_{2 M}}\right)$ is given by (I) we see that $a_{i_{2 M-1}}+h-q$ is an entry of $\left(\ldots, \hat{a}_{i_{2 M}-2}, \hat{a}_{i_{2 M}-1}, \hat{a}_{i_{2 M}}\right)$. Using (b) in 1.3 we see that

$$
\left\{\ldots, \hat{a}_{i_{2 M}-2}, \hat{a}_{i_{2 M}-1}, \hat{a}_{i_{2 M}}\right\}=\left(\ldots, b_{i_{2 M}-2}, b_{i_{2 M}-1}, b_{i_{2 M}}\right)
$$

as multisets. We see that cases (III) and (IV) cannot arise. Hence we must be in case (I) or (II). Thus we have either

(a)

$$
\begin{aligned}
& \left(b_{i_{2 M-1}}, b_{i_{2 M-1}+1}, \ldots, b_{i_{2 M}-2}, b_{i_{2 M}-1}, b_{i_{2 M}}\right) \\
& =\left(a_{i_{2 M-1}}, a_{i_{2 M-1}+1}, \ldots, a_{i_{2 M}-2}, a_{i_{2 M}-1}, a_{i_{2 M}}\right)
\end{aligned}
$$

or

(b) $\left(b_{i_{2 M-1}}, b_{i_{2 M-1}+1}, \ldots, b_{i_{2 M}-2}, b_{i_{2 M}-1}, b_{i_{2 M}}\right)$

$$
=\left(a_{i_{2 M-1}+1}, a_{i_{2 M-1}}, a_{i_{2 M-1}+3}, a_{i_{2 M-1}+2}, \ldots, a_{i_{2 M}}, a_{i_{2 M}-1}\right) \text {. }
$$

1.5. Let $a^{\prime}=\left(a_{0}, a_{1}, a_{2}, \ldots, a_{i_{2 M-1}-1}\right), b^{\prime}=\left(b_{0}, b_{1}, b_{2}, \ldots, b_{i_{2 M-1}-1}\right)$,

$$
\begin{aligned}
& \hat{a}^{\prime}=\left(a_{0}, a_{1}+1, a_{2}+1, a_{3}+2, a_{4}+2, \ldots, a_{i_{2 M-1}-1}+\left(i_{2 M-1}-1\right) / 2\right), \\
& \hat{b}^{\prime}=\left(b_{0}, b_{1}+1, b_{2}+1, b_{3}+2, b_{4}+2, \ldots, b_{i_{2 M-1}-1}+\left(i_{2 M-1}-1\right) / 2\right),
\end{aligned}
$$

From $[\hat{b}]=[\hat{a}]$, (b) in 1.3 and (a)+(b) in 1.4 we see that the multiset formed by the entries of $\hat{a}^{\prime}$ coincides with the multiset formed by the entries of $\hat{b}^{\prime}$. Using 
the induction hypothesis we see that there exists $X^{\prime} \subset\{1,3, \ldots, 2 M-3\}$ such that $b^{\prime}=a^{\prime X^{\prime}}$ where $a^{\prime X^{\prime}}$ is defined in terms of $a^{\prime}, X^{\prime}$ in the same way as $a^{X}$ was defined (see 1.1) in terms of $a, X$. We set $X=X^{\prime}$ if we are in case (a) of 1.4 and $X=X^{\prime} \cup\{2 M-1\}$ if we are in case (b). Then we have $b=a^{X}$ (see again (a) and (b) in 1.4), as required. This completes the proof of 1.2 .

1.6. We shall use the notation of 1.1 . Let $\mathfrak{T}$ be the set of all unordered pairs $(\mathfrak{A}, \mathfrak{B})$ where $\mathfrak{A}, \mathfrak{B}$ are subsets of $\{0,1,2, \ldots\}$ and $\mathfrak{A} \cup \dot{B}=\left(a_{0}, a_{1}, a_{2}, \ldots, a_{N}\right)$ as multisets. For example, setting $\mathfrak{A}_{\varnothing}=\left(a_{0}, a_{2}, a_{4}, \ldots, a_{N}\right)$ and $\mathfrak{B}_{\varnothing}=\left(a_{1}, a_{3}, \ldots, a_{N-1}\right)$, we have $\left(\mathfrak{A}_{\varnothing}, \mathfrak{B}_{\varnothing}\right) \in \mathfrak{T}$. For any subset $\mathfrak{a}$ of $\mathscr{f}$ we consider

$$
\begin{aligned}
\mathfrak{A}_{\mathfrak{a}} & =\left((\mathscr{q}-\mathfrak{a}) \cap \mathfrak{A}_{\varnothing}\right) \cup\left(\mathfrak{a} \cap \mathfrak{B}_{\varnothing}\right) \cup\left(\mathfrak{A}_{\varnothing} \cap \mathfrak{B}_{\varnothing}\right), \\
\mathfrak{B}_{\mathfrak{a}} & =\left((\mathscr{q}-\mathfrak{a}) \cap \mathfrak{B}_{\varnothing}\right) \cup\left(\mathfrak{a} \cap \mathfrak{A}_{\varnothing}\right) \cup\left(\mathfrak{A}_{\varnothing} \cap \mathfrak{B}_{\varnothing}\right) .
\end{aligned}
$$

Then $\left(\mathfrak{A}_{\mathfrak{a}}, \mathfrak{B}_{\mathfrak{a}}\right) \in \mathfrak{T}$ and the map $\mathfrak{a} \mapsto\left(\mathfrak{A}_{\mathfrak{a}}, \mathfrak{B}_{\mathfrak{a}}\right)$ induces a bijection $\overline{\mathscr{P}}(\mathscr{F}) \leftrightarrow \mathfrak{T}$. (Note that if $\mathfrak{a}=\varnothing$ then $\left(\mathfrak{A}_{\mathfrak{a}}, \mathfrak{B}_{\mathfrak{a}}\right)$ agrees with the earlier definition of $\left(\mathfrak{A}_{\varnothing}, \mathfrak{B}_{\varnothing}\right)$.)

Let $\mathfrak{T}^{\prime}$ be the set of all $(\mathfrak{A}, \mathfrak{B}) \in \mathfrak{T}$ such that $|\mathfrak{A}|=\left|\mathfrak{A}_{\varnothing}\right|$ and $|\mathfrak{B}|=\left|\mathfrak{B}_{\varnothing}\right|$.

Let $\mathscr{P}(\mathscr{F})_{0}$ be the subspace of $\mathscr{P}_{\mathrm{ev}}(\mathscr{F})$ spanned by the 2-element subsets

$$
\left\{a_{i_{0}}, a_{i_{1}}\right\},\left\{a_{i_{2}}, a_{i_{3}}\right\}, \ldots,\left\{a_{i_{2 M-2}}, a_{i_{2 M-1}}\right\}
$$

of $\mathscr{F}$. Let $\mathscr{P}(\mathscr{F})_{1}$ be the subspace of $\mathscr{P}_{\mathrm{ev}}(\mathscr{F})$ spanned by the 2 -element subsets

$$
\left\{a_{i_{1}}, a_{i_{2}}\right\},\left\{a_{i_{3}}, a_{i_{4}}\right\}, \ldots,\left\{a_{i_{2 M-1}}, a_{i_{2 M}}\right\}
$$

of $\mathscr{g}$.

Let $\overline{\mathscr{P}}(\mathscr{F})_{0}$ and $\overline{\mathscr{P}}(\mathscr{F})_{1}$ be the images of $\mathscr{P}(\mathscr{F})_{0}$ and $\mathscr{P}(\mathscr{F})_{1}$ under the obvious map $\mathscr{P}(\mathscr{F}) \rightarrow \overline{\mathscr{P}}(\mathscr{F})$. Then:

(a) $\overline{\mathscr{P}}(\mathscr{F})_{0}$ and $\overline{\mathscr{P}}(\mathscr{F})_{1}$ are opposed Lagrangian subspaces of the symplectic vector space $\overline{\mathscr{P}}(\mathscr{F}),($, ) (see 0.7); hence ( , ) defines an identification

$$
\overline{\mathscr{P}}(\mathscr{F})_{0}=\overline{\mathscr{P}}(\mathscr{F})_{1}^{*},
$$

where $\overline{\mathscr{P}}(\mathscr{F})_{1}^{*}$ is the vector space dual to $\overline{\mathscr{P}}(\mathscr{F})_{1}$.

Let $\mathfrak{T}_{0}$ and $\mathfrak{T}_{1}$ be the subsets of $\mathfrak{T}$ corresponding to $\overline{\mathscr{P}}(\mathscr{F})_{0}$ and $\overline{\mathscr{P}}(\mathscr{F})_{1}$, respectively, under the bijection $\overline{\mathscr{P}}(\mathscr{g}) \leftrightarrow \mathfrak{T}$. Note that $\mathfrak{T}_{0} \subset \mathfrak{T}^{\prime}, \mathfrak{T}_{1} \subset \mathfrak{T}^{\prime}$, and $\left|\mathfrak{T}_{0}\right|=\left|\mathfrak{T}_{1}\right|=2^{M}$.

For any $X \subset\{1,3, \ldots, 2 M-1\}$ we set $\mathfrak{a}_{X}=\bigcup_{s \in X}\left\{a_{i_{s}}, a_{i_{s+1}}\right\} \in \mathscr{P}(\mathscr{F})$. Then $\left(\mathfrak{A}_{\mathfrak{a}_{X}}, \mathfrak{B}_{\mathfrak{a}_{X}}\right) \in \mathfrak{T}_{1}$ is related to $a^{X}$ in 1.1 as follows:

$$
\mathfrak{A}_{\mathfrak{a}_{X}}=\left\{a_{0}^{X}, a_{2}^{X}, a_{4}^{X}, \ldots, a_{N}^{X}\right\}, \quad \mathfrak{B}_{\mathfrak{a}_{X}}=\left\{a_{1}^{X}, a_{3}^{X}, \ldots, a_{N-1}^{X}\right\} .
$$


1.7. We shall use the notation of 1.1 . Let $T$ be the set of all ordered pairs $(A, B)$ where $A$ is a subset of $\{0,1,2, \ldots\}, B$ is a subset of $\{1,2,3, \ldots\}, A$ contains no consecutive integers, $B$ contains no consecutive integers, and $A \dot{\cup} B=$ $\left(\hat{a}_{0}, \hat{a}_{1}, \hat{a}_{2}, \ldots, \hat{a}_{N}\right)$ as multisets. For example, setting $A_{\varnothing}=\left(\hat{a}_{0}, \hat{a}_{2}, \hat{a}_{4}, \ldots, \hat{a}_{N}\right)$ and $B_{\varnothing}=\left(\hat{a}_{1}, \hat{a}_{3}, \ldots, \hat{a}_{N-1}\right)$, we have $\left(A_{\varnothing}, B_{\varnothing}\right) \in T$.

For any $(A, B) \in T$ we define $\left(A^{-}, B^{-}\right)$as follows: $A^{-}$consists of $x_{0}<x_{1}-1<$ $x_{2}-2<\cdots<x_{p}-p$ where $x_{0}<x_{1}<\cdots<x_{p}$ are the elements of $A ; B^{-}$consists of $y_{1}-1<y_{2}-2<\cdots<y_{q}-q$ where $y_{1}<y_{2}<\cdots<y_{q}$ are the elements of $B$.

We can enumerate the elements of $T$ as in [Lusztig 1984b, 11.5]. Let $J$ be the set of all $c \in \mathbb{N}$ such that $c$ appears exactly once in the sequence

$\left(\hat{a}_{0}, \hat{a}_{1}, \hat{a}_{2}, \ldots, \hat{a}_{N}\right)=\left(a_{0}, a_{1}+1, a_{2}+1, a_{3}+2, a_{4}+2, \ldots, a_{N-1}+N / 2, a_{N}+N / 2\right)$.

A nonempty subset $I$ of $J$ is said to be an interval if it is of the form $\{i, i+1$, $i+2, \ldots, j\}$ with $i-1 \notin J, j+1 \notin J$ and with $i \neq 0$. Let $\mathscr{I}$ be the set of intervals of $J$. For any $s \in\{1,3, \ldots, 2 M-1\}$, the set $I_{s}:=\left\{\hat{a}_{i_{s}}, \hat{a}_{i_{s}+1}, \hat{a}_{i_{s}+2}, \ldots, \hat{a}_{i_{s}+2 m_{s}+1}\right\}$ is either a single interval or a union of intervals $I_{s}^{1} \sqcup I_{s}^{2} \sqcup \ldots \sqcup I_{s}^{t_{s}}\left(t_{s} \geq 2\right)$ where $\hat{a}_{i_{s}} \in I_{s}^{1}, \hat{a}_{i_{s}+2 m_{s}+1} \in I_{s}^{t_{s}},\left|I_{s}^{1}\right|,\left|I_{s}^{t_{s}}\right|$ are odd, $\left|I_{s}^{h}\right|$ are even for $h \in\left[2, t_{s}-1\right]$ and any element in $I_{s}^{e}$ is $<$ than any element in $I_{s}^{e^{\prime}}$ for $e<e^{\prime}$. Let $\Phi_{s}$ be the set of all $I \in \mathscr{I}$ such that $I \subset I_{s}$. Let $H$ be the set of all $c \in J$ such that $c$ does not belong to any interval. For any subset $\alpha \subset \mathscr{I}$ we consider

$$
\begin{aligned}
& A_{\alpha}=\bigcup_{I \in \mathscr{I}-\alpha}\left(I \cap A_{\varnothing}\right) \cup \bigcup_{I \in \alpha}\left(I \cap B_{\varnothing}\right) \cup\left(H \cap A_{\varnothing}\right) \cup\left(A_{\varnothing} \cap B_{\varnothing}\right), \\
& B_{\alpha}=\bigcup_{I \in \mathscr{I}-\alpha}\left(I \cap B_{\varnothing}\right) \cup \bigcup_{I \in \alpha}\left(I \cap A_{\varnothing}\right) \cup\left(H \cap B_{\varnothing}\right) \cup\left(A_{\varnothing} \cap B_{\varnothing}\right) .
\end{aligned}
$$

Then $\left(A_{\alpha}, B_{\alpha}\right) \in T$ and the map $\alpha \mapsto\left(A_{\alpha}, B_{\alpha}\right)$ is a bijection $\mathscr{P}(\mathscr{I}) \leftrightarrow T$. (Note that if $\alpha=\varnothing$ then $\left(A_{\alpha}, B_{\alpha}\right)$ agrees with the earlier definition of $\left(A_{\varnothing}, B_{\varnothing}\right)$.)

Let $T^{\prime}=\left\{(A, B) \in T ;|A|=\left|A_{\varnothing}\right|,|B|=\left|B_{\varnothing}\right|\right\}, T_{1}=\left\{(A, B) \in T^{\prime} ; A^{-} \dot{\cup} B^{-}=\right.$ $\left.A_{\varnothing}^{-} \dot{\cup} B_{\varnothing}^{-}\right\}$. Let $\mathscr{P}(\mathscr{I})^{\prime}$ and $\mathscr{P}(\mathscr{I})_{1}$ be the subsets of $\mathscr{P}(\mathscr{I})$ corresponding to $T^{\prime}$ and $T_{1}$ under the bijection $\mathscr{P}(\mathscr{I}) \leftrightarrow T$.

Now let $X$ be a subset of $\{1,3, \ldots, 2 M-1\}$. Let $\alpha_{X}=\bigcup_{s \in X} \mathscr{I}_{s} \in \mathscr{P}(\mathscr{Y})$. From the definitions we see that

$$
A_{\alpha_{X}}^{-}=\mathfrak{A}_{\mathfrak{a}_{X}}, \quad B_{\alpha_{X}}^{-}=\mathfrak{B}_{\mathfrak{a}_{X}}
$$

(in the notation of 1.6). In particular we have $\left(A_{\alpha_{X}}, B_{\alpha_{X}}\right) \in T_{1}$. Thus $\left|T_{1}\right| \geq 2^{M}$. Using Lemma 1.2 we see that

$$
\left|T_{1}\right|=2^{M} \quad \text { and } \quad T_{1}=\left\{\left(A_{\alpha_{X}}, B_{\alpha_{X}}\right) ; X \subset\{1,3, \ldots, 2 M-1\}\right\} .
$$

Using (a) and (b) we deduce:

$$
\text { The map } T_{1} \rightarrow \mathfrak{T}_{1} \text { given by }(A, B) \mapsto\left(A^{-}, B^{-}\right) \text {is a bijection. }
$$




\section{Combinatorics (continued)}

2.1. Let $N \in \mathbb{N}$. Let

$$
a:=\left(a_{0}, a_{1}, a_{2}, \ldots, a_{N}\right) \in \mathbb{N}^{N+1}
$$

be such that $a_{0} \leq a_{1} \leq a_{2} \leq \cdots \leq a_{N}, a_{0}<a_{2}<a_{4}<\cdots, a_{1}<a_{3}<a_{5}<\cdots$ and such that the set $\mathscr{F}:=\left\{i \in[0, N] ; a_{i}\right.$ appears exactly once in $\left.a\right\}$ is nonempty. Now $\mathscr{F}$ consists of $\mu+1$ elements $i_{0}<i_{1}<\cdots<i_{\mu}$, where $\mu \in \mathbb{N}, \mu=N \bmod 2$. We have $i_{s}=s \bmod 2$ for $s \in[0, \mu]$. Hence for any $s \in[0, \mu-1]$ we have $i_{s+1}=i_{s}+2 m_{s}+1$ for some $m_{s} \in \mathbb{N}$. Let $\mathscr{E}$ be the set of $b:=\left(b_{0}, b_{1}, b_{2}, \ldots, b_{N}\right) \in \mathbb{N}^{N+1}$ such that $b_{0}<b_{2}<b_{4}<\cdots, b_{1}<b_{3}<b_{5}<\cdots$ and such that $[b]=[a]$ (we denote by $[b],[a]$ the multisets $\left.\left\{b_{0}, b_{1}, \ldots, b_{N}\right\},\left\{a_{0}, a_{1}, \ldots, a_{N}\right\}\right)$. We have $a \in \mathscr{E}$. For $b \in \mathscr{E}$ we set

$$
\stackrel{\circ}{b}=\left(\stackrel{\circ}{b}_{0}, \stackrel{\circ}{b}_{1}, \stackrel{\circ}{b}_{2}, \ldots, \stackrel{\circ}{b}_{N}\right)=\left(b_{0}, b_{1}, b_{2}+1, b_{3}+1, b_{4}+2, b_{5}+2, \ldots\right) \in \mathbb{N}^{N+1} .
$$

Let $[\stackrel{\circ}{b}]$ be the multiset $\left\{\stackrel{\circ}{b}_{0}, \stackrel{\circ}{b}_{1}, \stackrel{\circ}{b}_{2}, \ldots, \stackrel{\circ}{b}_{N}\right\}$. For any $s \in[0, \mu-1] \in 2 \mathbb{N}$ we define $a^{\{s\}}=\left(a_{0}^{\{s\}}, a_{1}^{\{s\}}, a_{2}^{\{s\}}, \ldots, a_{N}^{\{s\}}\right) \in \mathscr{E}$ by

$$
\begin{array}{r}
\left(a_{i_{s}}^{\{s\}}, a_{i_{s}+1}^{\{s\}}, a_{i_{s}+2}^{\{s\}}, a_{i_{s}+3}^{\{s\}}, \ldots, a_{i_{s}+2 m_{s}}^{\{s\}}, a_{i_{s}+2 m_{s}+1}^{\{s\}}\right) \\
\quad=\left(a_{i_{s}+1}, a_{i_{s}}, a_{i_{s}+3}, a_{i_{s}+2}, \ldots, a_{i_{s}+2 m_{s}+1}, a_{i_{s}+2 m_{s}}\right)
\end{array}
$$

and $a_{i}^{\{s\}}=a_{i}$ if $i \in[0, N]-\left[i_{s}, i_{s+1}\right]$. More generally, for a subset $X$ of $[0, \mu-1] \cap 2 \mathbb{N}$ we define $a^{X}=\left(a_{0}^{X}, a_{1}^{X}, a_{2}^{X}, \ldots, a_{N}^{X}\right) \in \mathscr{E}$ by $a_{i}^{X}=a_{i}^{\{s\}}$ if $s \in X, i \in\left[i_{s}, i_{s+1}\right]$, and $a_{i}^{X}=a_{i}$ for all other $i \in[0, N]$. Note that $\left[\stackrel{\circ}{a}^{X}\right]=[\stackrel{\circ}{a}]$. Conversely:

Lemma 2.2. Let $b \in \mathscr{E}$ be such that $[\stackrel{\circ}{b}]=[\stackrel{a}{a}]$. Then there exists $X \subset[0, \mu-1] \cap 2 \mathbb{N}$ such that $b=a^{X}$.

The proof is given in 2.3-2.5.

2.3. We argue by induction on $\mu$. By the argument in 1.3 we have

$$
\begin{array}{ll}
b_{i}=a_{i} & \text { for } i<i_{0}, \\
b_{i}=a_{i} & \text { for } i>i_{\mu} .
\end{array}
$$

If $\mu=0$ we see that $b=a$ and there is nothing further to prove. In the rest of the proof we assume that $\mu \geq 1$.

2.4. From 2.3 we see that $\left(a_{i_{0}}, a_{i_{0}+1}, \ldots, a_{N}\right)=\left(a_{i_{0}}<x_{1}=x_{1}<x_{2}=x_{2}<\cdots<\right.$ $\left.x_{p}=x_{p}<a_{i_{1}}<\ldots\right)$ (for some $p$ ) has the same entries as $\left(b_{i_{0}}, b_{i_{0}+1}, \ldots, b_{N}\right)$ (in some order). Hence the pair $\left(b_{i_{0}}, b_{i_{0}+2}, b_{i_{0}+4}, \ldots\right),\left(b_{i_{0}+1}, b_{i_{0}+3}, b_{i_{0}+5}, \ldots\right)$ must have one of the following four forms.

$$
\left(a_{i_{0}}, x_{1}, x_{2}, \ldots, x_{p}, \ldots\right),\left(x_{1}, x_{2}, \ldots, x_{p}, a_{i_{1}}, \ldots\right),
$$




$$
\begin{aligned}
& \left(x_{1}, x_{2}, \ldots, x_{p}, a_{i_{1}}, \ldots\right),\left(a_{i_{0}}, x_{1}, x_{2}, \ldots, x_{p}, \ldots\right), \\
& \left(a_{i_{0}}, x_{1}, x_{2}, \ldots, x_{p}, a_{i_{1}}, \ldots\right),\left(x_{1}, x_{2}, \ldots, x_{p}, \ldots\right), \\
& \left(x_{1}, x_{2}, \ldots, x_{p}, \ldots\right),\left(a_{i_{0}}, x_{1}, x_{2}, \ldots, x_{p}, a_{i_{1}}, \ldots\right) .
\end{aligned}
$$

Hence $\left(b_{i_{0}}, b_{i_{0}+1}, b_{i_{0}+2}, \ldots, b_{N}\right)$ must have one of the following four forms.

(I) $\left(a_{i_{0}}, x_{1}, x_{1}, x_{2}, x_{2}, \ldots, x_{p}, x_{p}, a_{i_{1}}, \ldots\right)$,

(II) $\left(x_{1}, a_{i_{0}}, x_{2}, x_{1}, x_{3}, x_{2}, \ldots, x_{p}, x_{p-1}, a_{i_{1}}, x_{p}, \ldots\right)$,

(III) $\left(a_{i_{0}}, x_{1}, x_{1}, x_{2}, x_{2}, \ldots, x_{p}, x_{p}, z, a_{i_{1}}, \ldots\right)$,

(IV) $\left(x_{1}, a_{i_{0}}, x_{2}, x_{1}, x_{3}, x_{2}, \ldots, x_{p}, x_{p-1}, z^{\prime}, x_{p}, z^{\prime \prime}, a_{i_{1}}, \ldots\right)$,

where $a_{i_{1}}<z, a_{i_{1}}<z^{\prime} \leq z^{\prime \prime}$ and all entries in ... are $>a_{i_{1}}$. Correspondingly, $\left(\stackrel{\circ}{b}_{i_{0}},{\stackrel{\circ}{i_{0}+1}}_{i_{1}},{\stackrel{\circ}{b_{0}+2}}_{i_{0}}, \ldots, \stackrel{\circ}{b}_{N}\right)$ must have one of the following four forms.

(I) $\left(a_{i_{0}}+h, x_{1}+h, x_{1}+h+1, x_{2}+h+1, x_{2}+h+2, \ldots, x_{p}+h+p-1\right.$, $\left.x_{p}+h+p, a_{i_{1}}+h+p, \ldots\right)$,

(II) $\left(x_{1}+h, a_{i_{0}}+h, x_{2}+h+1, x_{1}+h+1, x_{3}+h+2, x_{2}+h+2, \ldots, x_{p}+h+p-1\right.$, $\left.x_{p-1}+h+p-1, a_{i_{1}}+h+p, x_{p}+h+p, \ldots\right)$,

(III) $\left(a_{i_{0}}+h, x_{1}+h, x_{1}+h+1, x_{2}+h+1, x_{2}+h+2, \ldots, x_{p}+h+p-1, x_{p}+h+p\right.$, $\left.z+p, a_{i_{1}}+h+p+1, \ldots\right)$,

(IV) $\left(x_{1}+h, a_{i_{0}}+h, x_{2}+h+1, x_{1}+h+1, x_{3}+h+2, x_{2}+h+2, \ldots, x_{p}+h+p-1\right.$, $\left.x_{p-1}+h+p-1, z^{\prime}+h+p, x_{p}+h+p, z^{\prime \prime}+h+p+1, a_{i_{1}}+h+p+1, \ldots\right)$,

where $h=i_{0} / 2$ and in cases (III) and (IV) $a_{i_{1}}+h+p$ is not an entry of $\left({\stackrel{\circ}{b_{0}}}_{i_{0}},{\stackrel{\circ}{i_{0}+1}}_{1}\right.$, \left.${\stackrel{\circ}{b_{0}+2}}_{i}, \ldots\right)$.

Since $\left(\stackrel{\circ}{a}_{i_{0}}, \stackrel{\circ}{a}_{i_{0}+1}, \stackrel{\circ}{a}_{i_{0}+2}, \ldots\right)$ is given by (I) we see that $a_{i_{1}}+h+p$ is an entry of $\left(\stackrel{\circ}{a}_{i_{0}},{\stackrel{\circ}{i_{0}+1}}_{1}, \stackrel{\circ}{a}_{i_{0}+2}, \ldots\right)$. Using 2.3 we see that

$$
\left\{\stackrel{\circ}{a}_{i_{0}},{\stackrel{\circ}{i_{0}+1}}_{i_{0}},{\stackrel{\circ}{a_{0}+2}}_{0_{0}}, \ldots\right\}=\left\{\stackrel{\circ}{b}_{i_{0}},{\stackrel{\circ}{b_{0}+1}}_{i_{0}},{\stackrel{\circ}{b_{i}+2}}_{i_{0}}, \ldots\right\}
$$

as multisets. We see that cases (III) and (IV) cannot arise. Hence we must be in case (I) or (II). Thus we have either

$$
\left(b_{i_{0}}, b_{i_{0}+1}, b_{i_{0}+2}, \ldots, b_{i_{1}}\right)=\left(a_{i_{0}}, a_{i_{0}+1}, a_{i_{0}+2}, \ldots, a_{i_{1}}\right)
$$

or

(b) $\quad\left(b_{i_{0}}, b_{i_{0}+1}, b_{i_{0}+2}, \ldots, b_{i_{1}}\right)=\left(a_{i_{0}+1}, a_{i_{0}}, a_{i_{0}+3}, a_{i_{0}+2}, \ldots, a_{i_{1}}, a_{i_{1}-1}\right)$.

From 2.3 and (a)+(b) we see that if $\mu=1$ then Lemma 2.2 holds. Thus in the rest of the proof we can assume that $\mu \geq 2$. 
2.5. Let $a^{\prime}=\left(a_{i_{1}+1}, a_{i_{1}+2}, \ldots, a_{N}\right), b^{\prime}=\left(b_{i_{1}+1}, b_{i_{1}+2}, \ldots, b_{N}\right)$,

$$
\begin{aligned}
& \stackrel{\circ}{a}^{\prime}=\left(a_{i_{1}+1}, a_{i_{1}+2}, a_{i_{1}+3}+1, a_{i_{1}+4}+1, a_{i_{1}+5}+2, a_{i_{1}+6}+2, \ldots\right), \\
& \stackrel{\circ}{b}^{\prime}=\left(b_{i_{1}+1}, b_{i_{1}+2}, b_{i_{1}+3}+1, b_{i_{1}+4}+1, b_{i_{1}+5}+2, b_{i_{1}+6}+2, \ldots\right) .
\end{aligned}
$$

From $[\stackrel{\circ}{b}]=[\stackrel{\circ}{a}]$, (a) in 2.3 and (a)+(b) in 2.4 we see that the multiset formed by the entries of $\stackrel{\circ}{a}^{\prime}$ coincides with the multiset formed by the entries of $\dot{b}^{\prime}$. Using the induction hypothesis we see that there exists $X^{\prime} \subset[2, \mu-1] \cap 2 \mathbb{N}$ such that $b^{\prime}=a^{\prime X^{\prime}}$ where $a^{\prime X^{\prime}}$ is defined in terms of $a^{\prime}, X^{\prime}$ in the same way as $a^{X}$ (see 2.1) was defined in terms of $a, X$. We set $X=X^{\prime}$ if we are in case (a) of 2.4 and $X=\{0\} \cup X^{\prime}$ if we are in case (b). Then we have $b=a^{X}$ (see again (a) and (b) of 2.4), as required. This completes the proof of Lemma 2.2.

2.6. We shall use the notation of 2.1. Let $\mathfrak{T}$ be the set of all unordered pairs $(\mathfrak{A}, \mathfrak{B})$ where $\mathfrak{A}, \mathfrak{B}$ are subsets of $\{0,1,2, \ldots\}$ and $\mathfrak{A} \dot{\cup} \mathfrak{B}=\left(a_{0}, a_{1}, a_{2}, \ldots, a_{N}\right)$ as multisets. For example, setting

$$
\mathfrak{A}_{\varnothing}=\left\{a_{i} ; i \in[0, N] \cap 2 \mathbb{N}\right\} \quad \text { and } \quad \mathfrak{B}_{\varnothing}=\left\{a_{i} ; i \in[0, N] \cap(2 \mathbb{N}+1)\right\} \text {, }
$$

we have $\left(\mathfrak{A}_{\varnothing}, \mathfrak{B}_{\varnothing}\right) \in \mathfrak{T}$. For any subset $\mathfrak{a}$ of $\mathscr{g}$ we consider

$$
\begin{aligned}
\mathfrak{A}_{\mathfrak{a}} & =\left((\mathscr{g}-\mathfrak{a}) \cap \mathfrak{A}_{\varnothing}\right) \cup\left(\mathfrak{a} \cap \mathfrak{B}_{\varnothing}\right) \cup\left(\mathfrak{A}_{\varnothing} \cap \mathfrak{B}_{\varnothing}\right), \\
\mathfrak{B}_{\mathfrak{a}} & =\left((\mathscr{q}-\mathfrak{a}) \cap \mathfrak{B}_{\varnothing}\right) \cup\left(\mathfrak{a} \cap \mathfrak{A}_{\varnothing}\right) \cup\left(\mathfrak{A}_{\varnothing} \cap \mathfrak{B}_{\varnothing}\right) .
\end{aligned}
$$

Then $\left(\mathfrak{A}_{\mathfrak{a}}, \mathfrak{B}_{\mathfrak{a}}\right)=\left(\mathfrak{A}_{\mathscr{q}-\mathfrak{a}}, \mathfrak{A}_{\mathscr{g}-\mathfrak{a}}\right) \in \mathfrak{T}$ and the map $\mathfrak{a} \mapsto\left(\mathfrak{A}_{\mathfrak{a}}, \mathfrak{B}_{\mathfrak{a}}\right)$ induces a bijection $\overline{\mathscr{P}}(\mathscr{F}) \leftrightarrow \mathfrak{T}$. (Note that if $\mathfrak{a}=\varnothing$ then $\left(\mathfrak{A}_{\mathfrak{a}}, \mathfrak{B}_{\mathfrak{a}}\right)$ agrees with the earlier definition of $\left(\mathfrak{A}_{\varnothing}, \mathfrak{B}_{\varnothing}\right)$.)

Let $\mathfrak{T}^{\prime}$ be the set of all $(\mathfrak{A}, \mathfrak{B}) \in \mathfrak{T}$ such that $|\mathfrak{A}|=\left|\mathfrak{A}_{\varnothing}\right|$ and $|\mathfrak{B}|=\left|\mathfrak{B}_{\varnothing}\right|$. Let $\mathscr{P}(\mathscr{F})_{1}$ be the subspace of $\mathscr{P}(\mathscr{F})$ spanned by the following 2-element subsets of $\mathscr{F}$ :

$$
\begin{array}{ll}
\left\{a_{i_{1}}, a_{i_{2}}\right\},\left\{a_{i_{3}}, a_{i_{4}}\right\}, \ldots,\left\{a_{i_{\mu-2}}, a_{i_{\mu-1}}\right\} & \text { if } N \text { is odd, } \\
\left\{a_{i_{1}}, a_{i_{2}}\right\},\left\{a_{i_{3}}, a_{i_{4}}\right\}, \ldots,\left\{a_{i_{\mu-1}}, a_{i_{\mu}}\right\} & \text { if } N \text { is even. }
\end{array}
$$

Let $\mathscr{P}(\mathscr{F})_{0}$ be the subspace of $\mathscr{P}(\mathscr{F})$ spanned by the following 2-element subsets of $\mathscr{F}$ :

$$
\begin{array}{ll}
\left\{a_{i_{0}}, a_{i_{1}}\right\},\left\{a_{i_{2}}, a_{i_{3}}\right\}, \ldots,\left\{a_{i_{\mu-1}}, a_{i_{\mu}}\right\} & \text { if } N \text { is odd, } \\
\left\{a_{i_{0}}, a_{i_{1}}\right\},\left\{a_{i_{2}}, a_{i_{3}}\right\}, \ldots,\left\{a_{i_{\mu-2}}, a_{i_{\mu-1}}\right\} & \text { if } N \text { is even. }
\end{array}
$$

Let $\overline{\mathscr{P}}(\mathscr{F})_{0}$ and $\overline{\mathscr{P}}(\mathscr{F})_{1}$ be the images of $\mathscr{P}(\mathscr{F})_{0}$ and $\mathscr{P}(\mathscr{F})_{1}$ under the obvious map $\mathscr{P}(\mathscr{F}) \rightarrow \overline{\mathscr{P}}(\mathscr{F})$. Then:

(a) $\overline{\mathscr{P}}(\mathscr{F})_{0}$ and $\overline{\mathscr{P}}(\mathscr{F})_{1}$ are opposed Lagrangian subspaces of the symplectic vector space $\overline{\mathscr{P}}_{\mathrm{ev}}(\mathscr{F}),\left(\right.$, ) (see 0.7); hence ( , ) defines an identification $\overline{\mathscr{P}}(\mathscr{F})_{1}=$ $\overline{\mathscr{P}}(\mathscr{F})_{0}^{*}$, where $\overline{\mathscr{P}}(\mathscr{F})_{0}^{*}$ is the vector space dual to $\overline{\mathscr{P}}(\mathscr{F})_{0}$. 
Let $\mathfrak{T}_{0}$ and $\mathfrak{T}_{1}$ be the subsets of $\mathfrak{T}$ corresponding to $\overline{\mathscr{P}}(\mathscr{F})_{0}$ and $\overline{\mathscr{P}}(\mathscr{F})_{1}$ under the bijection $\overline{\mathscr{P}}(\mathscr{F}) \leftrightarrow \mathfrak{T}$. Note that $\mathfrak{T}_{0} \subset \mathfrak{T}^{\prime}, \mathfrak{T}_{1} \subset \mathfrak{T}^{\prime},\left|\mathfrak{T}_{0}\right|=\left|\mathfrak{T}_{1}\right|=2^{\lfloor\mu / 2\rfloor}$.

For any $X \subset[0, \mu-1] \cap 2 \mathbb{N}$ we set $\mathfrak{a}_{X}=\bigcup_{s \in X}\left\{a_{i_{s}}, a_{i_{s+1}}\right\} \in \mathscr{P}(\mathscr{F})$. Then $\left(\mathfrak{A}_{\mathfrak{a}_{X}}, \mathfrak{B}_{\mathfrak{a}_{X}}\right)$ is related to $a^{X}$ in 2.1 as follows:

$$
\mathfrak{A}_{\mathfrak{a}_{X}}=\left\{a_{i}^{X} ; i \in[0, N] \cap 2 \mathbb{N}\right\}, \mathfrak{B}_{\mathfrak{a}_{X}}=\left\{a_{i}^{X} ; i \in[0, N] \cap(2 \mathbb{N}+1)\right\} .
$$

2.7. We shall use the notation of 2.1 . Let $T$ be the set of all unordered pairs $(A, B)$ where $A$ is a subset of $\{0,1,2, \ldots\}, B$ is a subset of $\{1,2,3, \ldots\}, A$ contains no consecutive integers, $B$ contains no consecutive integers, and $A \dot{\cup} B=$ $\left(\stackrel{\circ}{a}_{0}, \stackrel{\circ}{a}_{1}, \stackrel{\circ}{a}_{2}, \ldots, \stackrel{\circ}{a}_{N}\right)$ as multisets. For example, setting

$$
A_{\varnothing}=\left\{\stackrel{\circ}{a}_{i} ; i \in[0, N] \cap 2 \mathbb{N}\right\} \quad \text { and } \quad B_{\varnothing}=\left(\stackrel{\circ}{a}_{i} ; i \in[0, N] \cap(2 \mathbb{N}+1)\right\},
$$

we have $\left(A_{\varnothing}, B_{\varnothing}\right) \in T$.

For any $(A, B) \in T$ we define $\left(A^{-}, B^{-}\right)$as follows: $A^{-}$consists of $x_{1}<x_{2}-1<$ $x_{3}-2<\cdots<x_{p}-p+1$ where $x_{1}<x_{2}<\cdots<x_{p}$ are the elements of $A ; B^{-}$ consists of $y_{1}<y_{2}-1<\cdots<y_{q}-q+1$ where $y_{1}<y_{2}<\cdots<y_{q}$ are the elements of $B$.

We can enumerate the elements of $T$ as in [Lusztig 1984b, 11.5]. Let $J$ be the set of all $c \in \mathbb{N}$ such that $c$ appears exactly once in the sequence

$$
\left(\stackrel{\circ}{a}_{0}, \stackrel{\circ}{a}_{1}, \stackrel{\circ}{a}_{2}, \ldots, \stackrel{\circ}{a}_{N}\right)=\left(a_{0}, a_{1}, a_{2}+1, a_{3}+1, a_{4}+2, a_{5}+2, \ldots\right) .
$$

A nonempty subset $I$ of $J$ is said to be an interval if it is of the form $\{i, i+1$, $i+2, \ldots, j\}$ with $i-1 \notin J, j+1 \notin J$. Let $\mathscr{I}$ be the set of intervals of $J$. For

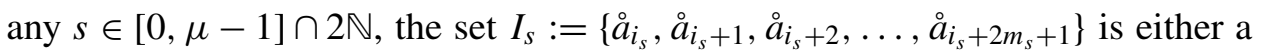
single interval or a union of intervals $I_{s}^{1} \sqcup I_{s}^{2} \sqcup \ldots \sqcup I_{s}^{t_{s}}\left(t_{s} \geq 2\right)$ where ${\stackrel{\circ}{i_{s}}}_{i_{s}} \in I_{s}^{1}$, $\stackrel{\circ}{a}_{i_{s}+2 m_{s}+1} \in I_{s}^{t_{s}},\left|I_{s}^{1}\right|,\left|I_{s}^{t_{s}}\right|$ are odd, $\left|I_{s}^{h}\right|$ are even for $h \in\left[2, t_{s}-1\right]$ and any element in $I_{s}^{e}$ is $<$ than any element in $I_{s}^{e^{\prime}}$ for $e<e^{\prime}$. Let $\Phi_{s}$ be the set of all $I \in \mathscr{I}$ such that $I \subset I_{s}$. For any subset $\alpha \subset \mathscr{I}$ we consider

$$
\begin{aligned}
& A_{\alpha}=\bigcup_{I \in \mathscr{I}-\alpha}\left(I \cap A_{\varnothing}\right) \cup \bigcup_{I \in \alpha}\left(I \cap B_{\varnothing}\right) \cup\left(A_{\varnothing} \cap B_{\varnothing}\right), \\
& B_{\alpha}=\bigcup_{I \in \mathscr{I}-\alpha}\left(I \cap B_{\varnothing}\right) \cup \bigcup_{I \in \alpha}\left(I \cap A_{\varnothing}\right) \cup\left(A_{\varnothing} \cap B_{\varnothing}\right) .
\end{aligned}
$$

Then $\left(A_{\alpha}, B_{\alpha}\right) \in T$ and the map $\alpha \mapsto\left(A_{\alpha}, B_{\alpha}\right)$ is a bijection $\overline{\mathscr{P}}(\mathscr{\Phi}) \leftrightarrow T$. (Note that if $\alpha=\varnothing$ then $\left(A_{\alpha}, B_{\alpha}\right)$ agrees with the earlier definition of $\left(A_{\varnothing}, B_{\varnothing}\right)$.)

Let

$$
\begin{aligned}
& T^{\prime}=\left\{(A, B) \in T ;|A|=\left|A_{\varnothing}\right|,|B|=\left|B_{\varnothing}\right|\right\}, \\
& T_{1}=\left\{(A, B) \in T^{\prime} ; A^{-} \dot{\cup} B^{-}=A_{\varnothing}^{-} \dot{\cup} B_{\varnothing}^{-}\right\} .
\end{aligned}
$$

Let $\overline{\mathscr{P}}(\mathscr{\Psi})^{\prime}$ and $\overline{\mathscr{P}}(\mathscr{I})_{1}$ be the subsets of $\overline{\mathscr{P}}(\mathscr{F})$ corresponding to $T^{\prime}$ and $T_{1}$ under the bijection $\overline{\mathscr{P}}(\mathscr{I}) \leftrightarrow T$. 

Now let $X$ be a subset of $[0, \mu-1] \cap 2 \mathbb{N}$. Let $\alpha_{X}=\bigcup_{s \in X} \mathscr{\Phi}_{s} \in \mathscr{P}(\mathscr{Y})$. From the
definitions we see that

$$
A_{\alpha_{X}}^{-}=\mathfrak{A}_{\mathfrak{a}_{X}}, \quad B_{\alpha_{X}}^{-}=\mathfrak{B}_{\mathfrak{a}_{X}}
$$

(in the notation of 2.6). In particular we have $\left(A_{\alpha_{X}}, B_{\alpha_{X}}\right) \in T_{1}$. Thus $\left|T_{1}\right| \geq 2^{\lfloor\mu / 2\rfloor}$. Using Lemma 2.2 we see that

$$
\left|T_{1}\right|=2^{\lfloor\mu / 2\rfloor} \quad \text { and } \quad T_{1}=\left\{\left(A_{\alpha_{X}}, B_{\alpha_{X}}\right) ; X \subset[0, \mu-1] \cap 2 \mathbb{N}\right\} .
$$

Using (a) and (b) we deduce:

$$
\text { The map } T_{1} \rightarrow \mathfrak{T}_{1} \text { given by }(A, B) \mapsto\left(A^{-}, B^{-}\right) \text {is a bijection. }
$$

\section{Proof of Theorem 0.4 and of Corollary 0.5}

3.1. If $G$ is simple adjoint of type $A_{n}, n \geq 1$, then Theorem 0.4 and Corollary 0.5 are obvious: we have $A(u)=\{1\}, \bar{A}(u)=\{1\}$.

3.2. Assume that $G=S p_{2 n}(\mathbb{k})$ where $n \geq 2$. Let $N$ be a sufficiently large even integer. Now $u: \mathbb{k}^{2 n} \rightarrow \mathbb{k}^{2 n}$ has $i_{e}$ Jordan blocks of size $e(e=1,2,3, \ldots)$. Here $i_{1}, i_{3}, i_{5}, \ldots$ are even. Let $\Delta=\left\{e \in\{2,4,6, \ldots\} ; i_{e} \geq 1\right\}$. Then $A(u)$ can be identified in the standard way with $\mathscr{P}(\Delta)$. Hence the group of characters $\hat{A}(u)$ of $A(u)$ (which may be canonically identified with the $F_{2}$-vector space dual to $\mathscr{P}(\Delta)$ ) may be also canonically identified with $\mathscr{P}(\Delta)$ itself (so that the basis given by the one-element subsets of $\Delta$ is self-dual).

To the partition $1 i_{1}+2 i_{2}+3 i_{3}+\cdots$ of $2 n$ we associate a pair $(A, B)$ as in [Lusztig 1984b, 11.6] (with $N, 2 m$ replaced by $2 n, N)$. We have $A=\left(\hat{a}_{0}, \hat{a}_{2}, \hat{a}_{4}, \ldots, \hat{a}_{N}\right)$, $B=\left(\hat{a}_{1}, \hat{a}_{3}, \ldots, \hat{a}_{N-1}\right)$, where $\hat{a}_{0} \leq \hat{a}_{1} \leq \hat{a}_{2} \leq \cdots \leq \hat{a}_{N}$ is obtained from a sequence $a_{0} \leq a_{1} \leq a_{2} \leq \cdots \leq a_{N}$ as in 1.1. (Here we use that $C$ is special.) Now the definitions and results in Section 1 are applicable. As in [Lusztig 1984a, 4.5] the family $\mathscr{F}$ is in canonical bijection with $\mathfrak{T}^{\prime}$ in 1.6.

We arrange the intervals in $\mathscr{I}$ in increasing order $I_{(1)}, I_{(2)}, \ldots, I_{(f)}$ (any element in $I_{(1)}$ is smaller than any element in $I_{(2)}$, etc.). We arrange the elements of $\Delta$ in increasing order $e_{1}<e_{2}<\cdots<e_{f^{\prime}}$; then $f=f^{\prime}$ and we have a bijection $\Phi \leftrightarrow \Delta, I_{(h)} \leftrightarrow e_{h}$; moreover we have $\left|I_{(h)}\right|=i_{e_{h}}$ for $h \in[1, f]$; see [Lusztig 1984b, 11.6]. Using this bijection we see that $A(u)$ and $\hat{A}(u)$ are identified with the $F_{2}$-vector space $\mathscr{P}(\mathscr{I})$ with basis given by the one-element subsets of $\mathscr{I}$. Let $\pi: \mathscr{P}(\mathscr{Y}) \rightarrow \mathscr{P}(\mathscr{Y})_{1}^{*}$ (the dual of $\mathscr{P}(\mathscr{I})_{1}$ in 1.7) be the (surjective) $F_{2}$-linear map which to $X \subset \mathscr{I}$ associates the linear form $L \mapsto|X \cap L| \bmod 2$ on $\mathscr{P}(\mathscr{I})_{1}$. We will show that

$$
\text { ker } \pi=\mathscr{K}(u), \quad \text { with } \mathscr{K}(u) \text { as in } 0.1 \text {. }
$$


We identify $\operatorname{Irr}_{C} W$ with $T^{\prime}$ (see 1.7) via the restriction of the bijection in [Lusztig 1984b, (12.2.4)] (we also use the description of the Springer correspondence in [Lusztig 1984b, 12.3]). Under this identification the subset $\operatorname{Irr}_{C}^{*} W$ of $\operatorname{Irr}_{C} W$ becomes the subset $T_{1}$ (see 1.7) of $T^{\prime}$. Via the identification $\mathscr{P}(\mathscr{I})^{\prime} \leftrightarrow T^{\prime}$ in 1.7 and $\hat{A}(u) \leftrightarrow \mathscr{P}(\mathscr{I})$ (see above), the map $E \mapsto \mathscr{V}_{E}$ from $T^{\prime}$ to $\hat{A}(u)$ becomes the obvious imbedding $\mathscr{P}(\mathscr{P})^{\prime} \rightarrow \mathscr{P}(\mathscr{P})$ (we use again [Lusztig 1984b, 12.3]). By definition, $\mathscr{K}(u)$ is the set of all $X \in \mathscr{P}(\mathscr{P})$ such that for any $L \in \mathscr{P}(\mathscr{I})_{1}$ we have $|X \cap L|=0 \bmod 2$. Thus, (a) holds.

Using (a) we have canonically $\bar{A}(u)=\mathscr{P}(\mathscr{I})_{1}^{*}$ via $\pi$. We define an $F_{2}$-linear map $\mathscr{P}(\mathscr{P})_{1} \rightarrow \overline{\mathscr{P}}(\mathscr{F})_{1}$ (see 1.6) by $I_{s} \mapsto\left\{a_{i_{s}}, a_{i_{s+1}}\right\}$ for $s \in\{1,3, \ldots, 2 M-1\}$ ( $I_{s}$ as in 1.7). This is an isomorphism; it corresponds to the bijection 1.7(c) under the identification $T_{1} \leftrightarrow \mathscr{P}(\mathscr{I})_{1}$ in 1.7 and the identification $\mathfrak{T}_{1} \leftrightarrow \overline{\mathscr{P}}(\mathscr{F})_{1}$ in 1.6. Hence we can identify $\mathscr{P}(\mathscr{Y})_{1}^{*}$ with $\overline{\mathscr{P}}(\mathscr{\mathscr { S }})_{1}^{*}$ and with $\overline{\mathscr{P}}(\mathscr{\mathscr { S }})_{0}$ (see $1.6(\mathrm{a})$ ). We obtain an identification $\bar{A}(u)=\overline{\mathscr{P}}(\mathscr{F})_{0}$.

By [Lusztig 1984a, 4.5] we have $\boldsymbol{X}_{\mathscr{F}}=\overline{\mathscr{P}}(\mathscr{F})$. Using 1.6(a) we see that $\overline{\mathscr{P}}(\mathscr{F})=$ $M\left(\overline{\mathscr{P}}(\mathscr{F})_{0}\right)=M(\bar{A}(u))$ canonically so that Theorem 0.4 holds in our case. From the arguments above we see that in our case Corollary 0.5 follows from 1.7(c).

3.3. Assume that $G=\mathrm{SO}_{n}(\mathbb{k})$ where $n \geq 7$. Let $N$ be a sufficiently large integer such that $N=n \bmod 2$. Now $u: \mathbb{k}^{n} \rightarrow \mathbb{k}^{n}$ has $i_{e}$ Jordan blocks of size $e(e=1,2,3, \ldots)$. Here $i_{2}, i_{4}, i_{6}, \ldots$ are even. Let $\Delta=\left\{e \in\{1,3,5, \ldots\} ; i_{e} \geq 1\right\}$. If $\Delta=\varnothing$ then $A(u)=\{1\}, \bar{A}(u)=\{1\}$ and $\mathscr{G}_{\mathscr{F}}=\{1\}$ so that the result is trivial.

In the remainder of this subsection we assume that $\Delta \neq \varnothing$. Then $A(u)$ can be identified in the standard way with the $F_{2}$-subspace $\mathscr{P}_{\mathrm{ev}}(\Delta)$ of $\mathscr{P}(\Delta)$ and the group of characters $\hat{A}(u)$ of $A(u)$ (which may be canonically identified with the $F_{2}$-vector space dual to $A(u)$ ) becomes $\overline{\mathscr{P}}(\Delta)$; the obvious pairing $A(u) \times \hat{A}(u) \rightarrow F_{2}$ is induced by the inner product $L, L^{\prime} \mapsto\left|L \cap L^{\prime}\right| \bmod 2$ on $\mathscr{P}(\Delta)$.

To the partition $1 i_{1}+2 i_{2}+3 i_{3}+\cdots$ of $n$ we associate a pair $(A, B)$ as in [Lusztig 1984b, 11.7] (with $N, M$ replaced by $n, N)$. We have $A=\left\{\stackrel{\circ}{a}_{i} ; i \in[0, N] \cap 2 \mathbb{N}\right\}$, $B=\left(\stackrel{\circ}{a}_{i} ; i \in[0, N] \cap(2 \mathbb{N}+1)\right\}$ where $\stackrel{\circ}{a}_{0} \leq \stackrel{\circ}{a}_{1} \leq \stackrel{\circ}{a}_{2} \leq \cdots \leq \stackrel{\circ}{a}_{N}$ is obtained from a sequence $a_{0} \leq a_{1} \leq a_{2} \leq \cdots \leq a_{N}$ as in 2.1. (Here we use that $C$ is special.) Now the definitions and results in $\$ 2$ are applicable. As in [Lusztig 1984a, 4.5] (if $N$ is even) or [Lusztig 1984a, 4.6] (if $N$ is odd) the family $\mathscr{F}$ is in canonical bijection with $\mathfrak{T}^{\prime}$ in 2.6.

We arrange the intervals in $\mathscr{I}$ in increasing order $I_{(1)}, I_{(2)}, \ldots, I_{(f)}$ (any element in $I_{(1)}$ is smaller than any element in $I_{(2)}$, etc.). We arrange the elements of $\Delta$ in increasing order $e_{1}<e_{2}<\cdots<e_{f^{\prime}}$; then $f=f^{\prime}$ and we have a bijection $\mathscr{I} \leftrightarrow \Delta, I_{(h)} \leftrightarrow e_{h}$; moreover we have $\left|I_{(h)}\right|=i_{e_{h}}$ for $h \in[1, f]$; see [Lusztig 1984b, 11.7]. Using this bijection we see that $A(u)$ is identified with $\mathscr{P}_{\mathrm{ev}}(\mathscr{I})$ and $\hat{A}(u)$ is identified with $\overline{\mathscr{P}}(\mathscr{I})$. For any $X \in \mathscr{P}_{\mathrm{ev}}(\mathscr{I})$, the assignment $L \mapsto|X \cap L| \bmod 2$ can 
be viewed as an element of $\overline{\mathscr{P}}(\mathscr{I})_{1}^{*}$ (the dual space of $\overline{\mathscr{P}}(\mathscr{I})_{1}$ in 2.7 which by 2.7 (b) is an $F_{2}$-vector space of dimension $2^{\lfloor\mu / 2\rfloor}$ ). This induces a (surjective) $F_{2}$-linear map $\pi: \mathscr{P}_{\mathrm{ev}}(\mathscr{Y}) \rightarrow \overline{\mathscr{P}}(\mathscr{F})_{1}^{*}$. We will show that

$$
\operatorname{ker} \pi=\mathscr{K}(u), \quad \text { with } \mathscr{K}(u) \text { as in } 0.1 \text {. }
$$

We identify $\operatorname{Irr}_{C} W$ with $T^{\prime}$ (see 2.7) via the restriction of the bijection in [Lusztig 1984b, (13.2.5)] if $N$ is odd or [ibid., (13.2.6)] if $N$ is even (we also use the description of the Springer correspondence in [Lusztig 1984b, 13.3]). Under this identification the subset $\operatorname{Irr}_{C}^{*} W$ of $\operatorname{Irr}_{C} W$ becomes the subset $T_{1}$ (see 2.7) of $T^{\prime}$. Via the identification $\overline{\mathscr{P}}(\mathscr{\mathscr { T }})^{\prime} \leftrightarrow T^{\prime}$ in 2.7 and $\hat{A}(u) \leftrightarrow \overline{\mathscr{P}}(\mathscr{I})$ (see above), the map $E \mapsto \mathscr{V}_{E}$ from $T^{\prime}$ to $\hat{A}(u)$ becomes the obvious imbedding $\overline{\mathscr{P}}(\mathscr{\mathscr { S }})_{0} \rightarrow \overline{\mathscr{P}}(\mathscr{\mathscr { \Phi }})$ (we use again [ibid., 13.3]). By definition, $\mathscr{K}(u)$ is the set of all $X \in \mathscr{P}_{\mathrm{ev}}(\mathscr{F})$ such that for any $L \in \mathscr{P}(\mathscr{I})$ representing a vector in $\overline{\mathscr{P}}(\mathscr{\mathscr { S }})_{1}$ we have $|X \cap L|=0$ mod 2 . Thus, (a) holds.

Using (a) we have canonically $\bar{A}(u)=\overline{\mathscr{P}}(\mathscr{\Phi})_{1}^{*}$ via $\pi$. We have an $F_{2}$-linear map $\overline{\mathscr{P}}(\mathscr{I})_{1} \rightarrow \overline{\mathscr{P}}(\mathscr{F})_{0}$ (see 2.6) induced by $I_{s} \mapsto\left\{a_{i_{s}}, a_{i_{s+1}}\right\}$ for $s \in[0, \mu-1] \cap 2 \mathbb{N}\left(I_{s}\right.$ as in 2.7). This is an isomorphism; it corresponds to the bijection 2.7(c) under the identification $T_{1} \leftrightarrow \overline{\mathscr{P}}(\mathscr{F})_{1}$ in 2.7 and the identification $\mathfrak{T}_{1} \leftrightarrow \overline{\mathscr{P}}(\mathscr{F})_{0}$ in 2.6. Hence we can identify $\overline{\mathscr{P}}(\mathscr{F})_{1}^{*}$ with $\overline{\mathscr{P}}(\mathscr{F})_{0}^{*}$ and with $\overline{\mathscr{P}}(\mathscr{F})_{1}$ (see 2.6(a)). We obtain an identification $\bar{A}(u)=\overline{\mathscr{P}}(\mathscr{\mathscr { S }})_{1}$.

By [Lusztig 1984a, 4.6] we have $\boldsymbol{X}_{\mathscr{F}}=\overline{\mathscr{P}}_{\mathrm{ev}}(\mathscr{F})$. Using 2.6 (a) we see that $\overline{\mathscr{P}}(\mathscr{F})=M\left(\overline{\mathscr{P}}(\mathscr{F})_{1}\right)=M(\bar{A}(u))$ canonically so that Theorem 0.4 holds in our case. From the arguments above we see that in our case Corollary 0.5 follows from 2.7(c).

3.4. In 3.5-3.9 we consider the case where $G$ is simple adjoint of exceptional type. In each case we list the elements of the $\operatorname{set} \operatorname{Irr}_{C} W$ for each special unipotent class $C$ of $G$; an element $e$ of $\operatorname{Irr}_{C} W-\operatorname{Irr}_{C}^{*} W$ is listed as $[e]$. (The notation for the various $C$ is as in [Spaltenstein 1985]; the notation for the objects of $\operatorname{Irr} W$ is as in [Spaltenstein 1985] (for type $E_{n}$ ) and as in [Lusztig 1984a, 4.10] for type $F_{4}$.) In each case the structure of $A(u), \bar{A}(u)$ (for $u \in C$ ) is indicated; here $S_{n}$ denotes the symmetric group in $n$ letters. The order in which we list the objects in $\operatorname{Irr}_{C} W$ corresponds to the following order of the irreducible representations of $A(u)=S_{n}$ :

$$
\begin{aligned}
& 1, \epsilon(n=2), \\
& 1, r, \epsilon\left(n=3, G \neq G_{2}\right), \\
& 1, r\left(n=3, G=G_{2}\right), \\
& 1, \lambda^{1}, \lambda^{2}, \sigma(n=4), \\
& 1, v, \lambda^{1}, v^{\prime}, \lambda^{2}, \lambda^{3}(n=5),
\end{aligned}
$$

in the notation of [Lusztig 1984a, 4.3]. Now Theorem 0.4 and Corollary 0.5 follow in our case from the tables in 3.5-3.9 and the definitions in [Lusztig 1984a, 4.8-4.13]. (In those tables $S_{n}$ is the symmetric group in $n$ letters.) 
3.5. Assume that $G$ is of type $E_{8}$.

$\operatorname{Irr}_{E_{8}} W=\left\{1_{0}\right\} ; A(u)=\{1\}, \bar{A}(u)=\{1\}$.

$\operatorname{Irr}_{E_{8}\left(a_{1}\right)} W=\left\{8_{1}\right\} ; A(u)=\{1\}, \bar{A}(u)=\{1\}$.

$\operatorname{Irr}_{E_{8}\left(a_{2}\right)} W=\left\{35_{2}\right\} ; A(u)=\{1\}, \bar{A}(u)=\{1\}$.

$\operatorname{Irr}_{E_{7} A_{1}} W=\left\{112_{3}, 28_{8}\right\} ; A(u)=S_{2}, \bar{A}(u)=S_{2}$.

$\operatorname{Irr}_{D_{8}} W=\left\{210_{4}, 160_{7}\right\} ; A(u)=S_{2}, \bar{A}(u)=S_{2}$.

$\operatorname{Irr}_{E_{7}\left(a_{1}\right) A_{1}} W=\left\{560_{5},\left[50_{8}\right]\right\} ; A(u)=S_{2}, \bar{A}(u)=\{1\}$.

$\operatorname{Irr}_{E_{7}\left(a_{1}\right)} W=\left\{567_{6}\right\} ; A(u)=\{1\}, \bar{A}(u)=\{1\}$.

$\operatorname{Irr}_{D_{8}\left(a_{1}\right)} W=\left\{700_{6}, 300_{8}\right\} ; A(u)=S_{2}, \bar{A}(u)=S_{2}$.

$\operatorname{Irr}_{E_{7}\left(a_{2}\right) A_{1}} W=\left\{1400_{7}, 10089,56_{19}\right\} ; A(u)=S_{3}, \bar{A}(u)=S_{3}$.

$\operatorname{Irr}_{A_{8}} W=\left\{1400_{8}, 1575_{10}, 350_{14}\right\} ; A(u)=S_{3}, \bar{A}(u)=S_{3}$.

$\operatorname{Irr}_{D_{7}\left(a_{1}\right)} W=\left\{3240_{9},\left[1050_{10}\right]\right\} ; A(u)=S_{2}, \bar{A}(u)=\{1\}$.

$\operatorname{Irr}_{D_{8}\left(a_{3}\right)} W=\left\{2240_{10},\left[175_{12}\right], 840_{13}\right\} ; A(u)=S_{3}, \bar{A}(u)=S_{2}$.

$\operatorname{Irr}_{D_{6} A_{1}} W=\left\{2268_{10}, 1296_{13}\right\} ; A(u)=S_{2}, \bar{A}(u)=S_{2}$.

$\operatorname{Irr}_{E_{6}\left(a_{1}\right) A_{1}} W=\left\{4096_{11}, 4096_{12}\right\} ; A(u)=S_{2}, \bar{A}(u)=S_{2}$.

$\operatorname{Irr}_{E_{6}} W=\left\{525_{12}\right\} ; A(u)=\{1\}, \bar{A}(u)=\{1\}$.

$\operatorname{Irr}_{D_{7}\left(a_{2}\right)} W=\left\{4200_{12}, 3360_{13}\right\} ; A(u)=S_{2}, \bar{A}(u)=S_{2}$.

$\operatorname{Irr}_{E_{6}\left(a_{1}\right)} W=\left\{2800_{13}, 2100_{16}\right\} ; A(u)=S_{2}, \bar{A}(u)=S_{2}$.

$\operatorname{Irr}_{D_{5} A_{2}} W=\left\{4536_{13},\left[840_{14}\right]\right\} ; A(u)=S_{2}, \bar{A}(u)=\{1\}$.

$\operatorname{Irr}_{D_{6}\left(a_{1}\right) A_{1}} W=\left\{6075_{14},\left[700_{16}\right]\right\} ; A(u)=S_{2}, \bar{A}(u)=\{1\}$.

$\operatorname{Irr}_{A_{6} A_{1}} W=\left\{2835_{14}\right\} ; A(u)=\{1\}, \bar{A}(u)=\{1\}$.

$\operatorname{Irr}_{A_{6}} W=\left\{4200_{15}\right\} ; A(u)=\{1\}, \bar{A}(u)=\{1\}$.

$\operatorname{Irr}_{D_{6}\left(a_{1}\right)} W=\left\{5600_{15}, 2400_{17}\right\} ; A(u)=S_{2}, \bar{A}(u)=S_{2}$.

$\operatorname{Irr}_{2 A_{4}} W=\left\{4480_{16}, 4536_{18}, 5670_{18}, 1400_{20}, 1680_{22}, 70_{32}\right\} ; A(u)=S_{5}, \bar{A}(u)=S_{5}$.

$\operatorname{Irr}_{D_{5}} W=\left\{2100_{20}\right\} ; A(u)=\{1\}, \bar{A}(u)=\{1\}$.

$\operatorname{Irr}_{\left(A_{5} A_{1}\right)^{\prime \prime}} W=\left\{5600_{21}, 2400_{23}\right\} ; A(u)=S_{2}, \bar{A}(u)=S_{2}$.

$\operatorname{Irr}_{D_{4} A_{2}} W=\left\{4200_{15},\left[168_{24}\right]\right\} ; A(u)=S_{2}, \bar{A}(u)=\{1\}$.

$\operatorname{Irr}_{A_{4} A_{2} A_{1}} W=\left\{2835_{22}\right\} ; A(u)=\{1\}, \bar{A}(u)=\{1\}$.

$\operatorname{Irr}_{A_{4} A_{2}} W=\left\{4536_{23}\right\} ; A(u)=\{1\}, \bar{A}(u)=\{1\}$.

$\operatorname{Irr}_{D_{5}\left(a_{1}\right)} W=\left\{2800_{25}, 2100_{28}\right\} ; A(u)=S_{2}, \bar{A}(u)=S_{2}$.

$\operatorname{Irr}_{A_{4} 2 A_{1}} W=\left\{4200_{24}, 3360_{25}\right\} ; A(u)=S_{2}, \bar{A}(u)=S_{2}$.

$\operatorname{Irr}_{D_{4}} W=\left\{525_{36}\right\} ; A(u)=\{1\}, \bar{A}(u)=\{1\}$.

$\operatorname{Irr}_{A_{4} A_{1}} W=\left\{4096_{26}, 4096_{27}\right\} ; A(u)=S_{2}, \bar{A}(u)=S_{2}$.

$\operatorname{Irr}_{A_{4}} W=\left\{2268_{30}, 1296_{33}\right\} ; A(u)=S_{2}, \bar{A}(u)=S_{2}$.

$\operatorname{Irr}_{D_{4}\left(a_{1}\right) A_{2}}=\left\{2240_{28}, 840_{31}\right\} ; A(u)=S_{2}, \bar{A}(u)=S_{2}$.

$\operatorname{Irr}_{A_{3} A_{2}} W=\left\{3240_{31},\left[972_{32}\right]\right\} ; A(u)=S_{2}, \bar{A}(u)=\{1\}$.

$\operatorname{Irr}_{D_{4}\left(a_{1}\right) A_{1}} W=\left\{1400_{32}, 1575_{34}, 350_{38}\right\} ; A(u)=S_{3}, \bar{A}(u)=S_{3}$.

$\operatorname{Irr}_{D_{4}\left(a_{1}\right)} W=\left\{1400_{37}, 1008_{39}, 56_{49}\right\} ; A(u)=S_{3}, \bar{A}(u)=S_{3}$. 
$\operatorname{Irr}_{2 A_{2}} W=\left\{700_{42}, 300_{44}\right\} ; A(u)=S_{2}, \bar{A}(u)=S_{2}$.

$\operatorname{Irr}_{A_{3}} W=\left\{567_{46}\right\} ; A(u)=\{1\}, \bar{A}(u)=\{1\}$.

$\operatorname{Irr}_{A_{2} 2 A_{1}} W=\left\{560_{47}\right\} ; A(u)=\{1\}, \bar{A}(u)=\{1\}$.

$\operatorname{Irr}_{A_{2} A_{1}} W=\left\{210_{52}, 160_{55}\right\} ; A(u)=S_{2}, \bar{A}(u)=S_{2}$.

$\operatorname{Irr}_{A_{2}} W=\left\{112_{63}, 28_{68}\right\} ; A(u)=S_{2}, \bar{A}(u)=S_{2}$.

$\operatorname{Irr}_{2 A_{1}} W=\left\{35_{74}\right\} ; A(u)=\{1\}, \bar{A}(u)=\{1\}$.

$\operatorname{Irr}_{A_{1}} W=\left\{8_{91}\right\} ; A(u)=\{1\}, \bar{A}(u)=\{1\}$.

$\operatorname{Irr}_{\varnothing} W=\left\{1_{120}\right\} ; A(u)=\{1\}, \bar{A}(u)=\{1\}$.

3.6. Assume that $G$ is adjoint of type $E_{7}$.

$\operatorname{Irr}_{E_{7}} W=\left\{1_{0}\right\} ; A(u)=\{1\}, \bar{A}(u)=\{1\}$.

$\operatorname{Irr}_{E_{7}\left(a_{1}\right)} W=\left\{7_{1}\right\} ; A(u)=\{1\}, \bar{A}(u)=\{1\}$.

$\operatorname{Irr}_{E_{7}\left(a_{2}\right)} W=\left\{27_{2}\right\} ; A(u)=\{1\}, \bar{A}(u)=\{1\}$.

$\operatorname{Irr}_{D_{6} A_{1}} W=\left\{56_{3}, 21_{6}\right\} ; A(u)=S_{2}, \bar{A}(u)=S_{2}$.

$\operatorname{Irr}_{E_{6}} W=\left\{21_{3}\right\} ; A(u)=\{1\}, \bar{A}(u)=\{1\}$.

$\operatorname{Irr}_{E_{6}\left(a_{1}\right)} W=\left\{120_{4}, 105_{5}\right\} ; A(u)=S_{2}, \bar{A}(u)=S_{2}$.

$\operatorname{Irr}_{D_{6}\left(a_{1}\right) A_{1}} W=\left\{189_{5},\left[15_{7}\right]\right\} ; A(u)=S_{2}, \bar{A}(u)=\{1\}$.

$\operatorname{Irr}_{D_{6}\left(a_{1}\right)} W=\left\{210_{6}\right\} ; A(u)=\{1\}, \bar{A}(u)=\{1\}$.

$\operatorname{Irr}_{A_{6}} W=\left\{105_{6}\right\} ; A(u)=\{1\}, \bar{A}(u)=\{1\}$.

$\operatorname{Irr}_{D_{5} A_{1}} W=\left\{168_{6}\right\} ; A(u)=\{1\}, \bar{A}(u)=\{1\}$.

$\operatorname{Irr}_{D_{5}} W=\left\{189_{7}\right\} ; A(u)=\{1\}, \bar{A}(u)=\{1\}$.

$\operatorname{Irr}_{D_{6}\left(a_{2}\right) A_{1}} W=\left\{315_{7}, 280_{9}, 35_{13}\right\} ; A(u)=S_{3}, \bar{A}(u)=S_{3}$.

$\operatorname{Irr}_{\left(A_{5} A_{1}\right)^{\prime}}=\left\{405_{8}, 189_{10}\right\} ; A(u)=S_{2}, \bar{A}(u)=S_{2}$.

$\operatorname{Irr}_{D_{5}\left(a_{1}\right) A_{1}} W=\left\{378_{9}\right\} ; A(u)=\{1\}, \bar{A}(u)=\{1\}$.

$\operatorname{Irr}_{A_{4} A_{2}} W=\left\{210_{10}\right\} ; A(u)=\{1\}, \bar{A}(u)=\{1\}$.

$\operatorname{Irr}_{D_{5}\left(a_{1}\right)} W=\left\{420_{10}, 336_{11}\right\} ; A(u)=S_{2}, \bar{A}(u)=S_{2}$.

$\operatorname{Irr}_{A_{5}^{\prime \prime}} W=\left\{105_{12}\right\} ; A(u)=\{1\}, \bar{A}(u)=\{1\}$.

$\operatorname{Irr}_{A_{4} A_{1}} W=\left\{512_{11}, 512_{12}\right\} ; A(u)=S_{2}, \bar{A}(u)=S_{2}$.

$\operatorname{Irr}_{D_{4}} W=\left\{105_{15}\right\} ; A(u)=\{1\}, \bar{A}(u)=\{1\}$.

$\operatorname{Irr}_{A_{4}} W=\left\{420_{13}, 336_{14}\right\} ; A(u)=S_{2}, \bar{A}(u)=S_{2}$.

$\operatorname{Irr}_{A_{3} A_{2} A_{1}} W=\left\{210_{13}\right\} ; A(u)=\{1\}, \bar{A}(u)=\{1\}$.

$\operatorname{Irr}_{A_{3} A_{2}} W=\left\{378_{14},\left[84_{15}\right]\right\} ; A(u)=S_{2}, \bar{A}(u)=\{1\}$.

$\operatorname{Irr}_{D_{4}\left(a_{1}\right) A_{1}} W=\left\{405_{15}, 189_{17}\right\} ; A(u)=S_{2}, \bar{A}(u)=S_{2}$.

$\operatorname{Irr}_{D_{4}\left(a_{1}\right)} W=\left\{315_{16}, 280_{18}, 35_{22}\right\} ; A(u)=S_{3}, \bar{A}(u)=S_{3}$.

$\operatorname{Irr}_{\left(A_{3} A_{1}\right)^{\prime \prime}} W=\left\{189_{20}\right\} ; A(u)=\{1\}, \bar{A}(u)=\{1\}$.

$\operatorname{Irr}_{2 A_{2}} W=\left\{168_{21}\right\} ; A(u)=\{1\}, \bar{A}(u)=\{1\}$.

$\operatorname{Irr}_{A_{2} 3 A_{1}} W=\left\{105_{21}\right\} ; A(u)=\{1\}, \bar{A}(u)=\{1\}$.

$\operatorname{Irr}_{A_{3}} W=\left\{210_{21}\right\} ; A(u)=\{1\}, \bar{A}(u)=\{1\}$. 


$$
\begin{aligned}
& \operatorname{Irr}_{A_{2} 2 A_{1}} W=\left\{189_{22}\right\} ; A(u)=\{1\}, \bar{A}(u)=\{1\} . \\
& \operatorname{Irr}_{A_{2} A_{1}} W=\left\{120_{25}, 105_{26}\right\} ; A(u)=S_{2}, \bar{A}(u)=S_{2} . \\
& \operatorname{Irr}_{3 A_{1}^{\prime \prime}} W=\left\{21_{36}\right\} ; A(u)=\{1\}, \bar{A}(u)=\{1\} . \\
& \operatorname{Irr}_{A_{2}} W=\left\{56_{30}, 21_{33}\right\} ; A(u)=S_{2}, \bar{A}(u)=S_{2} . \\
& \operatorname{Irr}_{2 A_{1}} W=\left\{27_{37}\right\} ; A(u)=\{1\}, \bar{A}(u)=\{1\} . \\
& \operatorname{Irr}_{A_{1}} W=\left\{7_{46}\right\} ; A(u)=\{1\}, \bar{A}(u)=\{1\} . \\
& \operatorname{Ir}_{\varnothing} W=\left\{1_{63}\right\} ; A(u)=\{1\}, \bar{A}(u)=\{1\} .
\end{aligned}
$$

3.7. Assume that $G$ is adjoint of type $E_{6}$.

$$
\begin{aligned}
& \operatorname{Irr}_{E_{6}} W=\left\{1_{0}\right\} ; A(u)=\{1\}, \bar{A}(u)=\{1\} . \\
& \operatorname{Irr}_{E_{6}\left(a_{1}\right)} W=\left\{6_{1}\right\} ; A(u)=\{1\}, \bar{A}(u)=\{1\} . \\
& \operatorname{Irr}_{D_{5}} W=\left\{20_{2}\right\} ; A(u)=\{1\}, \bar{A}(u)=\{1\} . \\
& \operatorname{Irr}_{A_{5} A_{1}} W=\left\{30_{3}, 15_{5}\right\} ; A(u)=S_{2}, \bar{A}(u)=S_{2} . \\
& \operatorname{Irr}_{D_{5}\left(a_{1}\right)} W=\left\{64_{4}\right\} ; A(u)=\{1\}, \bar{A}(u)=\{1\} . \\
& \operatorname{Irr}_{A_{4} A_{1}} W=\left\{60_{5}\right\} ; A(u)=\{1\}, \bar{A}(u)=\{1\} . \\
& \operatorname{Irr}_{A_{4}} W=\left\{81_{6}\right\} ; A(u)=\{1\}, \bar{A}(u)=\{1\} . \\
& \operatorname{Irr}_{D_{4}} W=\left\{24_{6}\right\} ; A(u)=\{1\}, \bar{A}(u)=\{1\} . \\
& \operatorname{Irr}_{D_{4}\left(a_{1}\right)} W=\left\{80_{7}, 90_{8}, 20_{10}\right\} ; A(u)=S_{3}, \bar{A}(u)=S_{3} . \\
& \operatorname{Irr}_{2 A_{2}} W=\left\{24_{12}\right\} ; A(u)=\{1\}, \bar{A}(u)=\{1\} . \\
& \operatorname{Irr}_{A_{3}} W=\left\{81_{10}\right\} ; A(u)=\{1\}, \bar{A}(u)=\{1\} . \\
& \operatorname{Irr}_{A_{2} 2 A_{1}} W=\left\{60_{11}\right\} ; A(u)=\{1\}, \bar{A}(u)=\{1\} . \\
& \operatorname{Irr}_{A_{2} A_{1}} W=\left\{64_{13}\right\} ; A(u)=\{1\}, \bar{A}(u)=\{1\} . \\
& \operatorname{Irr}_{A_{2}} W=\left\{30_{15}, 15_{17}\right\} ; A(u)=S_{2}, \bar{A}(u)=S_{2} . \\
& \operatorname{Irr}_{2 A_{1}} W=\left\{20_{20}\right\} ; A(u)=\{1\}, \bar{A}(u)=\{1\} . \\
& \operatorname{Irr}_{A_{1}} W=\left\{6_{25}\right\} ; A(u)=\{1\}, \bar{A}(u)=\{1\} . \\
& \operatorname{Irr}_{\varnothing} W=\left\{1_{36}\right\} ; A(u)=\{1\}, \bar{A}(u)=\{1\} .
\end{aligned}
$$

3.8. Assume that $G$ is of type $F_{4}$.

$$
\begin{aligned}
& \operatorname{Irr}_{F_{4}} W=\left\{1_{1}\right\} ; A(u)=\{1\}, \bar{A}(u)=\{1\} . \\
& \operatorname{Irr}_{F_{4}\left(a_{1}\right)} W=\left\{4_{2}, 2_{3}\right\} ; A(u)=S_{2}, \bar{A}(u)=S_{2} . \\
& \operatorname{Irr}_{F_{4}\left(a_{2}\right)} W=\left\{9_{1}\right\} ; A(u)=\{1\}, \bar{A}(u)=\{1\} . \\
& \operatorname{Irr}_{B_{3}} W=\left\{8_{1}\right\} ; A(u)=\{1\}, \bar{A}(u)=\{1\} . \\
& \operatorname{Irr}_{C_{3}} W=\left\{8_{3}\right\} ; A(u)=\{1\}, \bar{A}(u)=\{1\} . \\
& \operatorname{Irr}_{F_{4}\left(a_{3}\right)} W=\left\{12_{1}, 9_{3}, 6_{2}, 1_{3}\right\} ; A(u)=S_{4}, \bar{A}(u)=S_{4} . \\
& \operatorname{Irr}_{\tilde{A}_{2}} W=\left\{8_{2}\right\} ; A(u)=\{1\}, \bar{A}(u)=\{1\} . \\
& \operatorname{Irr}_{A_{2}} W=\left\{8_{4},\left[1_{2}\right]\right\} ; A(u)=S_{2}, \bar{A}(u)=\{1\} . \\
& \operatorname{Irr}_{A_{1} \tilde{A}_{1}} W=\left\{9_{4}\right\} ; A(u)=\{1\}, \bar{A}(u)=\{1\} . \\
& \operatorname{Irr}_{\tilde{A}_{1}} W=\left\{4_{5}, 2_{2}\right\} ; A(u)=S_{2}, \bar{A}(u)=S_{2} .
\end{aligned}
$$


$\operatorname{Irr}_{\varnothing} W=\left\{1_{4}\right\} ; A(u)=\{1\}, \bar{A}(u)=\{1\}$.

3.9. Assume that $G$ is of type $G_{2}$.

$\operatorname{Irr}_{G_{2}} W$ is the unit representation; $A(u)=\{1\}, \bar{A}(u)=\{1\}$.

$\operatorname{Irr}_{G_{2}\left(a_{1}\right)} W$ consists of the reflection representation and the one dimensional representation on which the reflection with respect to a long (resp. short) simple coroot acts nontrivially (resp. trivially); $A(u)=S_{3}, \bar{A}(u)=S_{3}$.

$\operatorname{Irr}_{\varnothing} W=\{\operatorname{sgn}\} ; A(u)=\{1\}, \bar{A}(u)=\{1\}$.

3.10. This completes the proof of Theorem 0.4 and that of Corollary 0.5 .

We note that the definition of $\mathscr{G}_{\mathscr{F}}$ given in [Lusztig 1984a] (for type $C_{n}, B_{n}$ ) is $\overline{\mathscr{P}}(\mathscr{F})_{1}$ (in the setup of 3.2) and $\overline{\mathscr{P}}(\mathscr{F})_{0}$ (in the setup of 3.3) which is noncanonically isomorphic to $\bar{A}(u)$, unlike the definition adopted here that is, $\overline{\mathscr{P}}(\mathscr{F})_{0}$ (in the setup of 3.2) and $\overline{\mathscr{P}}(\mathscr{F})_{1}$ (in the setup of 3.3) which makes $\mathscr{G}_{\mathscr{F}}$ canonically isomorphic to $\bar{A}(u)$.

\section{References}

[Alvis and Lusztig 1982] D. Alvis and G. Lusztig, "On Springer's correspondence for simple groups of type $E_{n}(n=6,7,8)$ ”, Math. Proc. Cambridge Philos. Soc. 92:1 (1982), 65-72. MR 83k:20040 Zbl 0492.20028

[Borho and MacPherson 1981] W. Borho and R. MacPherson, "Représentations des groupes de Weyl et homologie d'intersection pour les variétés nilpotentes", C. R. Acad. Sci. Paris Sér. I Math. 292:15 (1981), 707-710. MR 82f:14002 Zbl 0467.20036

[Losev and Ostrik 2012] I. Losev and V. Ostrik, "Classification of finite dimensional irreducible modules over $W$-algebras", preprint, 2012. arXiv 1202.6097

[Lusztig 1979a] G. Lusztig, "A class of irreducible representations of a Weyl group", Indag. Math. (Proc.) 82:1 (1979), 323-335. MR 81a:20052 Zbl 0435.20021

[Lusztig 1979b] G. Lusztig, "Unipotent representations of a finite Chevalley group of type $E_{8}$ ", Quart. J. Math. Oxford Ser. (2) 30:119 (1979), 315-338. MR 80j:20041 Zbl 0418.20038

[Lusztig 1984a] G. Lusztig, Characters of reductive groups over a finite field, Annals of Mathematics Studies 107, Princeton University Press, Princeton, NJ, 1984. MR 86j:20038 Zbl 0556.20033

[Lusztig 1984b] G. Lusztig, "Intersection cohomology complexes on a reductive group", Invent. Math. 75:2 (1984), 205-272. MR 86d:20050 Zbl 0547.20032

[Lusztig 1992] G. Lusztig, "A unipotent support for irreducible representations”, Adv. Math. 94:2 (1992), 139-179. MR 94a:20073 Zbl 0789.20042

[Shoji 1979a] T. Shoji, "On the Springer representations of the Weyl groups of classical algebraic groups”, Comm. Algebra 7:16 (1979), 1713-1745. MR 81h:20007a Zbl 0423.20042

[Shoji 1979b] T. Shoji, "Correction to 'On the Springer representations of the Weyl groups of classical algebraic groups”, Comm. Algebra 7:18 (1979), 2027-2033. MR 81h:20007b Zbl 0426.20028

[Shoji 1980] T. Shoji, "On the Springer representations of Chevalley groups of type $F_{4}$ ", Comm. Algebra 8:5 (1980), 409-440. MR 81b:20013 Zbl 0434.20026

[Spaltenstein 1982] N. Spaltenstein, “Appendix", Math. Proc. Cambridge Philos. Soc. 92:1 (1982), 73-78. MR 83k:20040 Zbl 0492.20028 
[Spaltenstein 1985] N. Spaltenstein, "On the generalized Springer correspondence for exceptional groups", pp. 317-338 in Algebraic groups and related topics (Kyoto/Nagoya, 1983), edited by R. Hotta, Adv. Stud. Pure Math. 6, North-Holland, Amsterdam, 1985. MR 87g:20072b Zbl 0574.20029

[Springer 1976] T. A. Springer, "Trigonometric sums, Green functions of finite groups and representations of Weyl groups”, Invent. Math. 36 (1976), 173-207. MR 56 \#491 Zbl 0374.20054

Received March 5, 2012. Revised May 11, 2012.

GEORGE LUSZTIG

DEPARTMENT OF MATHEMATICS

MAssachusetTs Institute of TEChNOLOGY

RoOM 2-276

CAMBRIDGE, MA 02139-4307

UNITED STATES

gyuri@math.mit.edu 


\title{
PACIFIC JOURNAL OF MATHEMATICS
}

\author{
msp.org/pjm
}

Founded in 1951 by E. F. Beckenbach (1906-1982) and F. Wolf (1904-1989)

\section{EDITORS}

V. S. Varadarajan (Managing Editor)

Department of Mathematics

University of California

Los Angeles, CA 90095-1555

pacific@math.ucla.edu

Paul Balmer

Department of Mathematics

University of California

Los Angeles, CA 90095-1555

balmer@math.ucla.edu

Daryl Cooper

Department of Mathematics

University of California

Santa Barbara, CA 93106-3080 cooper@math.ucsb.edu

Jiang-Hua $\mathrm{Lu}$

Department of Mathematics

Pokfulam Rd., Hong Kong jhlu@maths.hku.hk
The University of Hong Kong

Don Blasius

Department of Mathematics University of California

Los Angeles, CA 90095-1555

blasius@math.ucla.edu

Robert Finn

Department of Mathematics Stanford University

Stanford, CA 94305-2125

finn@math.stanford.edu

Sorin Popa

Department of Mathematics

University of California

Los Angeles, CA 90095-1555 popa@math.ucla.edu

Paul Yang

Department of Mathematics Princeton University

Princeton NJ 08544-1000

yang@math.princeton.edu

\section{PRODUCTION}

Silvio Levy, Scientific Editor, production@msp.org

\section{SUPPORTING INSTITUTIONS}

ACADEMIA SINICA, TAIPEI

CALIFORNIA INST. OF TECHNOLOGY

INST. DE MATEMÁTICA PURA E APLICADA

KEIO UNIVERSITY

MATH. SCIENCES RESEARCH INSTITUTE

NEW MEXICO STATE UNIV.

OREGON STATE UNIV.

\author{
STANFORD UNIVERSITY \\ UNIV. OF BRITISH COLUMBIA \\ UNIV. OF CALIFORNIA, BERKELEY \\ UNIV. OF CALIFORNIA, DAVIS \\ UNIV. OF CALIFORNIA, LOS ANGELES \\ UNIV. OF CALIFORNIA, RIVERSIDE \\ UNIV. OF CALIFORNIA, SAN DIEGO \\ UNIV. OF CALIF., SANTA BARBARA
}

\author{
Vyjayanthi Chari \\ Department of Mathematics \\ University of California \\ Riverside, CA 92521-0135 \\ chari@math.ucr.edu \\ Kefeng Liu \\ Department of Mathematics \\ University of California \\ Los Angeles, CA 90095-1555 \\ liu@math.ucla.edu \\ Jie Qing \\ Department of Mathematics \\ University of California \\ Santa Cruz, CA 95064 \\ qing@cats.ucsc.edu
}

These supporting institutions contribute to the cost of publication of this Journal, but they are not owners or publishers and have no responsibility for its contents or policies.

See inside back cover or msp.org/pjm for submission instructions.

The subscription price for 2014 is US \$410/year for the electronic version, and \$535/year for print and electronic.

Subscriptions, requests for back issues and changes of subscribers address should be sent to Pacific Journal of Mathematics, P.O. Box 4163, Berkeley, CA 94704-0163, U.S.A. The Pacific Journal of Mathematics is indexed by Mathematical Reviews, Zentralblatt MATH, PASCAL CNRS Index, Referativnyi Zhurnal, Current Mathematical Publications and Web of Knowledge (Science Citation Index).

The Pacific Journal of Mathematics (ISSN 0030-8730) at the University of California, c/o Department of Mathematics, 798 Evans Hall \#3840, Berkeley, CA 94720-3840, is published twelve times a year. Periodical rate postage paid at Berkeley, CA 94704, and additional mailing offices. POSTMASTER: send address changes to Pacific Journal of Mathematics, P.O. Box 4163, Berkeley, CA 94704-0163.

PJM peer review and production are managed by EditFLOW ${ }^{\circledR}$ from Mathematical Sciences Publishers.

\section{PUBLISHED BY}

mathematical sciences publishers

nonprofit scientific publishing

http://msp.org/

(C) 2014 Mathematical Sciences Publishers 


\section{PACIFIC JOURNAL OF MATHEMATICS}

Volume $267 \quad$ No. $2 \quad$ February 2014

Sums of squares in algebraic function fields over a complete discretely valued field

KARIM JOHANNES BECHER, DAVID GRIMM and JAN VAN GEEL

On the equivalence problem for toric contact structures on $\mathbf{S}^{\mathbf{3}}$-bundles over $S^{2}$

\section{Charles P. Boyer and Justin PATi}

An almost-Schur type lemma for symmetric $(2,0)$ tensors and applications

Xu CHENG

Algebraic invariants, mutation, and commensurability of link complements

ERIC CHESEBRO and JASON DEBLOIS

Taut foliations and the action of the fundamental group on leaf spaces and universal circles

YOSUKE KANO

A new monotone quantity along the inverse mean curvature flow in $\mathbb{R}^{n}$

KwoK-Kun Kwong and Pengzi Miao

Nonfibered L-space knots

TYE LIDMAN and LIAM WATSON

Families and Springer's correspondence

GEORGE LUSZTIG

Reflexive operator algebras on Banach spaces

Florence Merlevède, Costel Peligrad and Magda Peligrad Harer stability and orbifold cohomology

NiCOLA PAGANI

Spectra of product graphs and permanents of matrices over finite rings

LE ANH VINH

The concavity of the Gaussian curvature of the convex level sets of minimal surfaces with respect to the height

PEI-HE WANG 